\title{
Habiter ou « faire avec » le sable
}

Domestiquer l'espace et la matière en contexte saharien

Living or "dealing with" sand

\section{Sébastien Boulay}

\section{(2) OpenEdition}

Journals

Édition électronique

URL : https://journals.openedition.org/tc/7231

DOI : $10.4000 /$ tc. 7231

ISSN : 1952-420X

Éditeur

Éditions de l'EHESS

Édition imprimée

Date de publication : 15 décembre 2013

Pagination : 76-99

ISBN : 978-2-7351-1654-6

ISSN : 0248-6016

Référence électronique

Sébastien Boulay, «Habiter ou « faire avec » le sable », Techniques \& Culture [En ligne], 61 | 2013, mis en ligne le 15 décembre 2016, consulté le 29 septembre 2022. URL : http://journals.openedition.org/tc/ 7231 ; DOI : https://doi.org/10.4000/tc.7231 


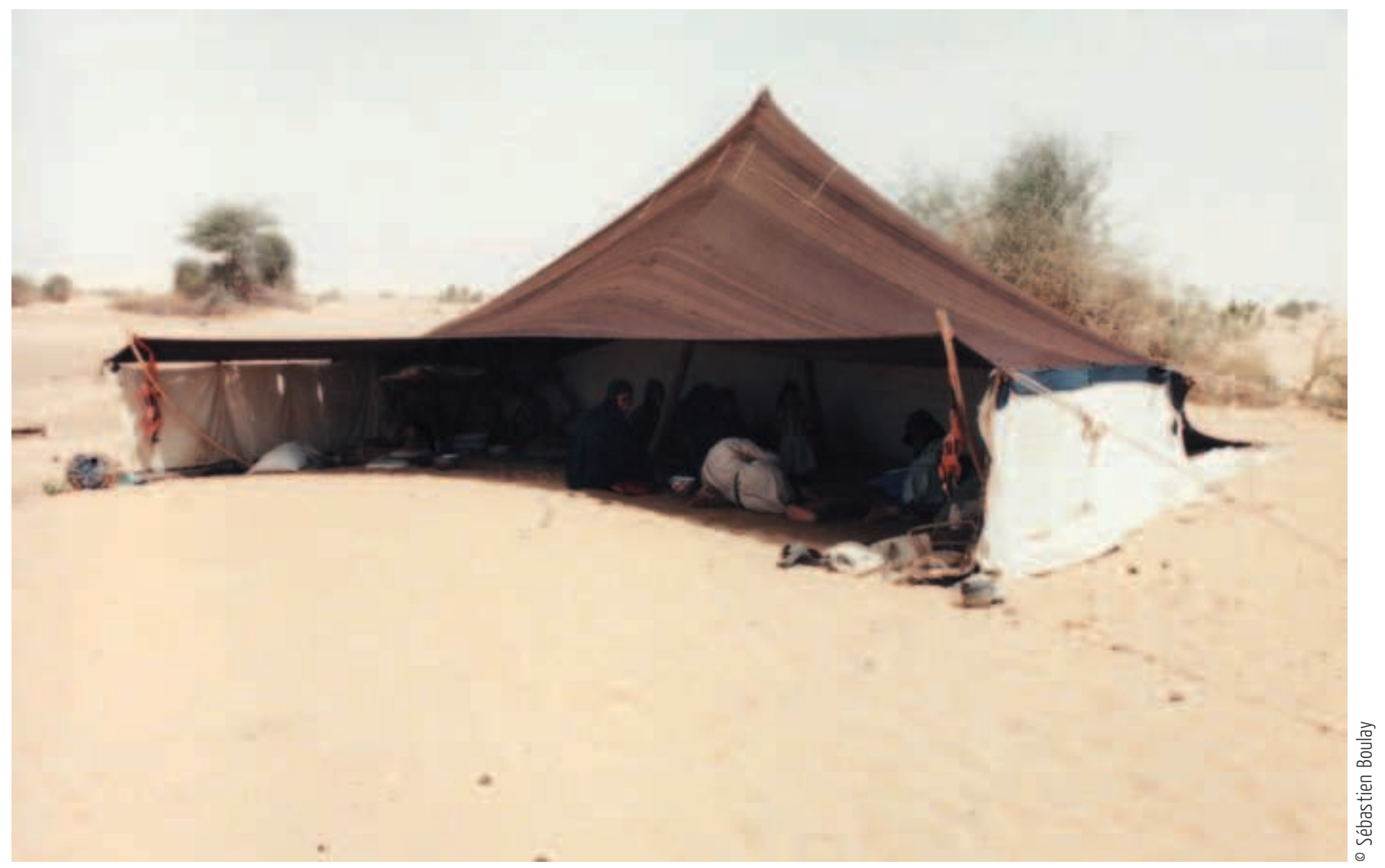




\section{HABITER OU « FAIRE AVEC » LE SABLE}

\section{Domestiquer l'espace et la matière en contexte saharien}

Nous souhaitons explorer dans ce texte les interactions les plus quotidiennes et routinières entre les Sahariens et le sable, matière omniprésente, tantôt pulvérulente et volatile, tantôt solide, tantôt souple voire liquide, qu'André Leroi-Gourhan avait classée en son temps parmi les « solides plastiques de faible cohésion» (1992 : 208), dont les seuls traitements applicables étaient selon lui « de les émietter, de les déplacer, de les tasser » (ibid. : 208). Le Sahara mauritanien et sa population actuelle constituent un terrain privilégié pour aborder cette question, notamment parce que cette partie du grand désert nord-africain présente la spécificité d'être essentiellement sableuse, ce qui n'est pas le cas d'autres régions du Sahara, souvent caillouteuses ou rocheuses.

Ce numéro spécial de Techniques \& culture nous offre ainsi l'opportunité de revenir sur des matériaux ethnographiques recueillis en 1999 et 2000 dans différentes régions de Mauritanie (Tagânt, Hodh, Trârza et Brâkna), sur la culture matérielle et domestique des pasteurs maures ou bidân* [cf. note de transcription en fin d'article] et sur ses mutations récentes dans un contexte de sédentarisation et d'urbanisation (Boulay 2003). Nous souhaitons retravailler ces matériaux à la lumière de ce nouveau questionnement sur le rapport dynamique des Sahariens au sable, et plus précisément sur les relations entre corps, artefacts et matière ambiante, cette dernière restant souvent oubliée des recherches anthropologiques sur les cultures matérielles.

Tellement présente dans le quotidien des pasteurs mauritaniens qu'ils en font rarement état, à l'exception d'allusions et de gestes quotidiens auxquels nul ne semble accorder d'importance, le chercheur a tendance à son tour à oublier le rôle déterminant de cette matière naturelle dans les modes de vie, l'histoire, la langue, l'économie, la cosmologie des 


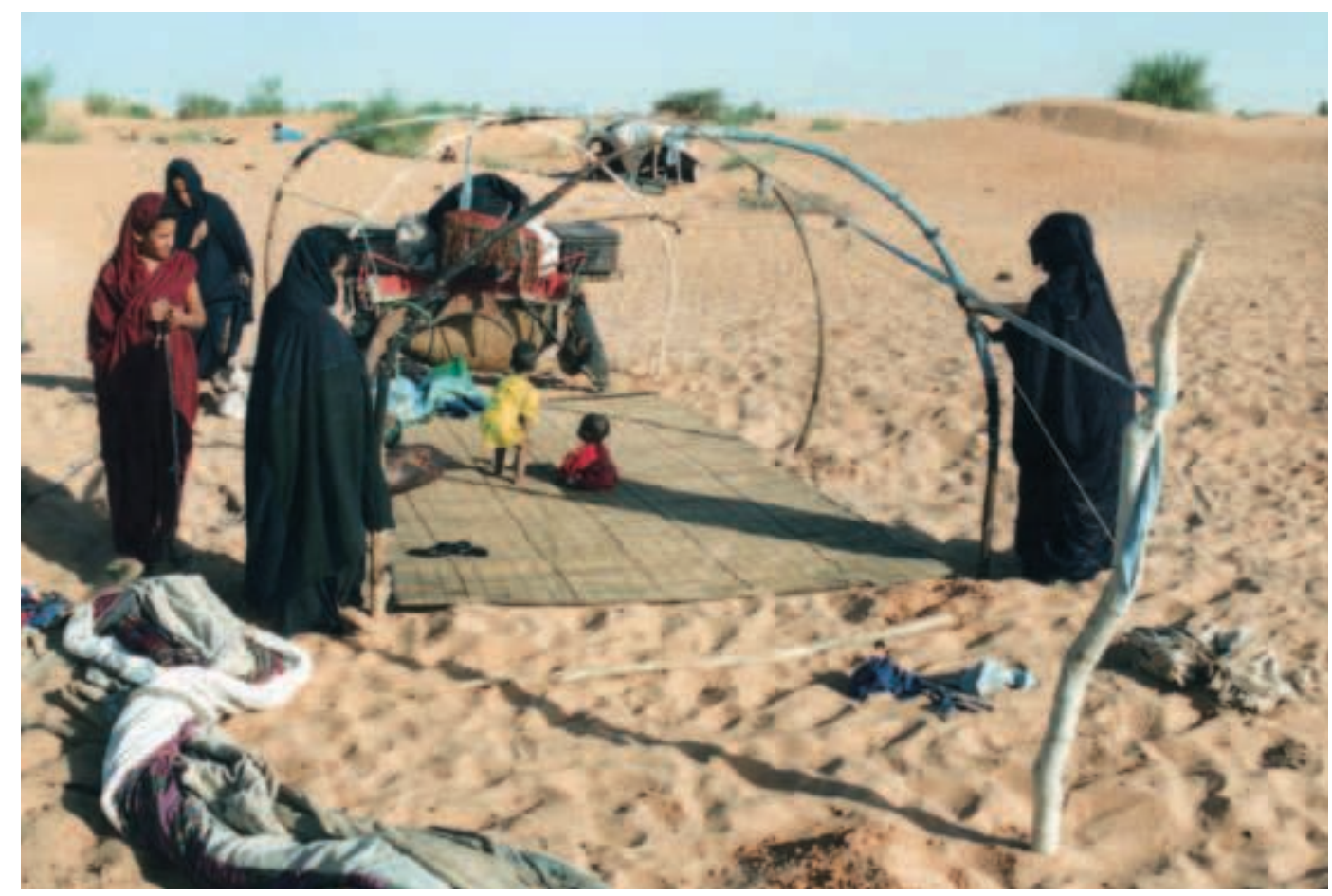

Structure à arceaux destinée à supporter un vélum de cotonnade importée habitants du désert. Le fait qu'une population fasse si peu de cas d'une matière pourtant omniprésente dans sa vie de tous les jours - comme on le fait si facilement de la multitude d'objets du quotidien qui nous entoure - peut effectivement conduire l'observateur à se désintéresser de la richesse des techniques grâce auxquelles elle parvient à la domestiquer dans sa quête quotidienne de confort.

Notre propos sera donc consacré aux interfaces les plus concrètes entre les habitants du désert mauritanien et le sable, médiatisées ou non par des objets, en l'occurrence celles qui se déroulent dans le lieu par excellence de la construction du sujet en société: l'espace domestique. Matérialisé au Sahara par la tente, ce dernier nous semble en effet le point d'observation le plus pertinent pour comprendre ce que « faire avec » le sable implique en termes de choix technologiques (Lemonnier 1993), d'ajustements moteurs et d'adaptations matérielles (ergonomie des objets).

Ce faisant, il s'agit de mettre au jour ce troisième protagoniste du « corps à corps » entre sujet et objet auquel le stimulant groupe de recherche « Matière à penser » s'est intéressé (Julien et Warnier 1999; Julien et Rosselin 2005), négligeant toutefois les matières (naturelles ou artificielles) ambiantes dans lesquelles sujets et objets interagissent. Si en effet ce corps à corps conduit le sujet à intégrer en permanence des objets et leurs dynamiques dans sa synthèse corporelle (Warnier 1999), cette incorporation prend également en compte - voire suppose un apprentissage préalable de - la matière ambiante dans laquelle elle s'opère et ses propriétés physico-chimiques, elles-mêmes changeantes : route sèche ou mouillée pour l'automobiliste, neige fraîche ou glacée pour le skieur, terre battue plus ou moins compacte pour la joueuse de tennis, mer plus ou moins formée pour le navigateur... sable déposé ou en suspension pour le Saharien. 
Dans l'ouest du Sahara, l'habitation consiste en un vélum de sept mètres par six environ, assemblage de bandes tissées dont les dimensions varient selon le statut social de la famille qu'elle abrite. Le vélum est le plus souvent supporté en son centre par les pointes de deux longues perches en bois (rkâyz) disposées obliquement, mais il peut aussi être tendu sur deux ou trois arceaux ('inâylîn) réalisés à partir de racines d'acacia ${ }^{1}$. Il s'agit par conséquent d'un espace très réduit au sein duquel vivent la famille conjugale et ses visiteurs, l'hospitalité étant dans cette société du désert une obligation incontournable. Un espace dépourvu de cloisons intérieures, où l'intimité n'est préservée que grâce à un code de pudeur (saḥwa) régissant les comportements socio-spatiaux et les attitudes interpersonnelles. La tente est le principal espace de la vie sociale en milieu bédouin saharien ${ }^{2}$.

Quelles contraintes pose le sable dans l'habiter et la vie domestique de ces familles? Comment intègrent-elles au quotidien les qualités et propriétés particulières du sable, avec quels critères de confort? Nous verrons en outre si cette omniprésence du sable donne lieu à des objets et à des savoir-faire spécifiques. Enfin, nous examinerons à quels réajustements de la relation au sable la sédentarisation et l'urbanisation ont pu éventuellement conduire.

\section{Représentations et gestion du sable dans l'invention de l'espace domestique}

Pour explorer cette interface, intéressonsnous en premier lieu à la valeur différentielle et au statut que les Maures assignent à cette matière ambiante, qui ne présente jamais, selon les lieux traversés et selon les saisons, les mêmes couleurs, les mêmes formes ni le même état. Cette diversité de natures du sable et de paysages offre une palette de possibilités aux habitants du désert, à partir desquelles ils vont produire des représentations, du lexique et des discours contrastés ${ }^{3}$.

Outre la double nécessité de proximité
Camp devenu insalubre et famille sur le départ

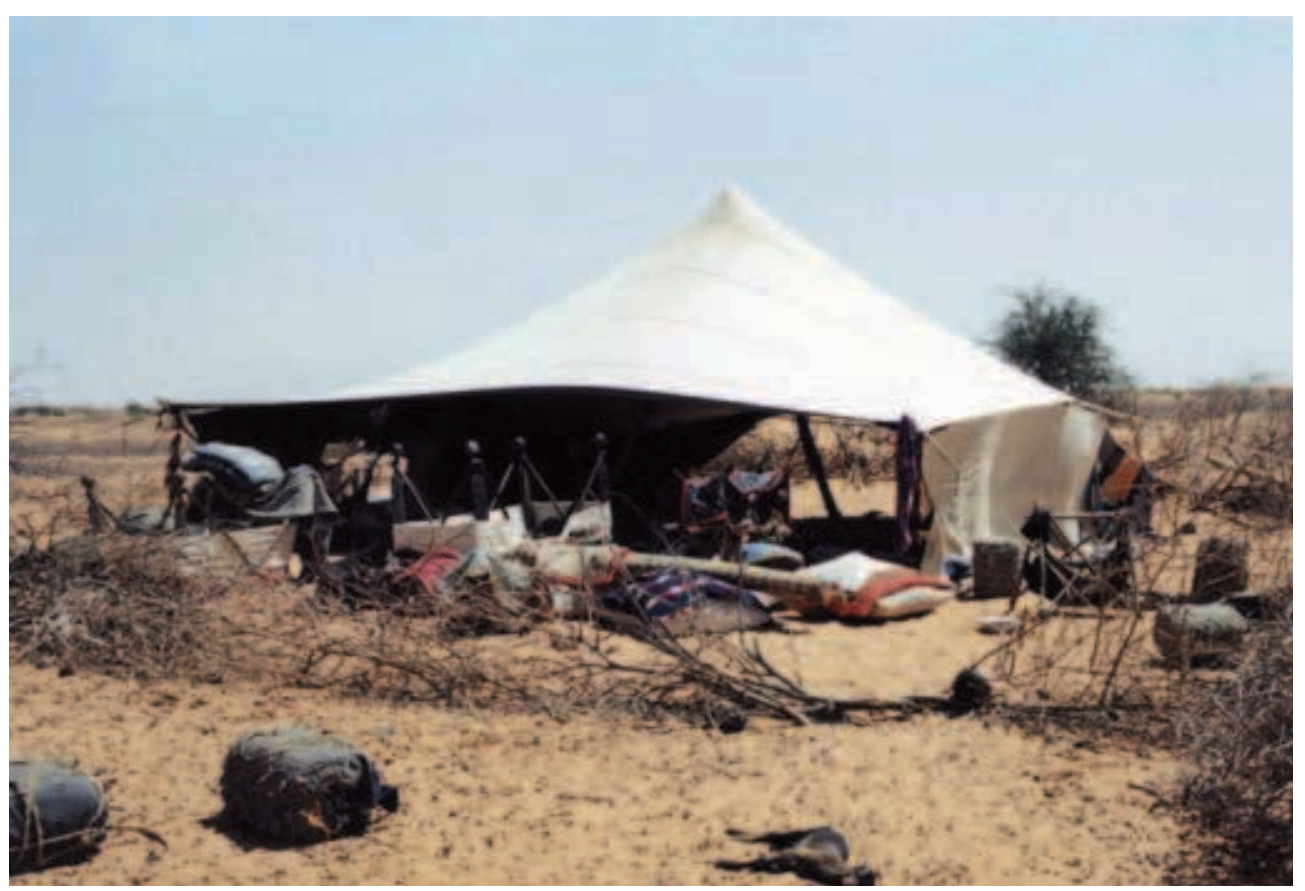




\section{Estrade utilisée par les pasteurs vivant dans des zones sahéliennes où le sable fait défaut}

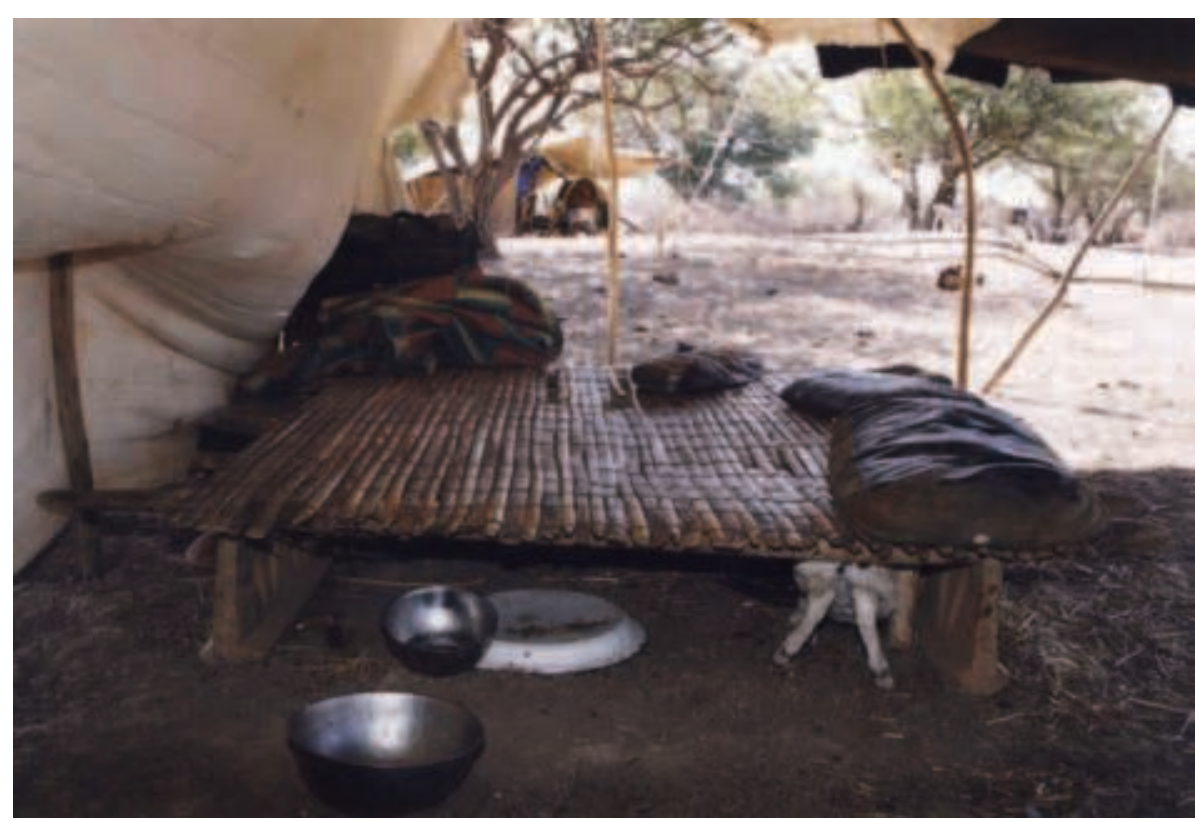

relative d'un point d'eau et de présence de végétation et de pâturages pour les animaux, la qualité du paysage et la nature du sol sont déterminantes dans le choix d'un site d'installation (dâr) et dans l'abandon d'un camp devenu insalubre. On va autant que possible rechercher un terrain de « terre blanche » (trâb al-bey $\underline{\partial} a$ ) autrement dit de sable, synonyme de pureté et de propreté, par opposition à la « terre noire » (trâb al-kahlla) ${ }^{4}$, expression renvoyant à un sable plus terreux que l'on retrouve près des dépressions ou dans les zones de transition avec le milieu sahélien. Le premier est qualifié de " propre » (maṣgûla) et associé à l'idée de confort (temânûkat), tandis que le second est considéré comme désagréable, salissant et peu indiqué pour la vie sous la tente. Les pasteurs maures qui vivent dans la partie sahélienne de la Mauritanie utilisent d'ailleurs systématiquement des estrades pour s'installer sous la tente, à l'abri de la terre, mais aussi des insectes et autres reptiles qui sont légion en particulier au moment de la saison des pluies (juin-septembre)

Ce sont bien les propriétés physico-chimiques de la « terre blanche » qui sont recherchées par les nomades: un sable qui est sec, souple et qui épouse les corps qui viennent s'y installer, un sable qui ne tache pas la peau ni le vêtement, bref qui ne salit pas, un sable qui absorbe facilement les liquides, qui se remplace ou se dame aisément, qui n'est ni trop pulvérulent ni trop compact. Le sable présente également l'avantage de rendre immédiatement visible tout corps étranger, liquide ou solide, toute impureté, et donc de se « nettoyer » aisément. L'endroit choisi pour déployer la natte de sol (hṣeyra) et au-dessus duquel on va ériger la tente, doit être au maximum débarrassé de tous les éléments considérés comme potentiellement nocifs au confort: brindilles, cailloux, touffes d'herbe sèche ou de cram-cram, protubérances sableuses. Car sous la tente on vit assis ou allongé à même la natte et on ressent parfaitement la moindre irrégularité du sol.

L'installation dans un « nouveau camp » (dâr jdîde) consiste symboliquement à s'approprier un espace considéré jusque-là comme habité par les djinns ${ }^{5}$ et suppose une série 
d'actes rituels qui impliquent un contact direct et tactile avec le sable. Lorsque les familles ont choisi un lieu pour s'établir et avant même que les bêtes n'aient été déchargées, le chef de famille implore la protection divine sur le nouveau terrain. Dans certaines familles religieuses, on prend une poignée de sable sous une touffe de paille ou sous un arbre, censée représenter la terre, et on pratique sur elle un « hjâ̂b», acte magique consistant dans ce cas à réciter la fâtiḥa (sourate liminaire du Coran) ainsi qu'une autre sourate, suivie d'un crachotement sur la poignée de sable, qui est enfin redéposée à sa place initiale. Ensuite, le nouveau terrain est envahi d'affaires et d'ustensiles de toutes sortes et la « maîtresse de tente » (mûlâ-t al-hayma) doit au plus vite dresser sa tente pour abriter les membres de sa famille, exténués par le voyage, la chaleur et la soif (Boulay 2005).

Survient alors un deuxième temps fort: une fois le rectangle de toile déployé sur le sol et les cordes de la tente étendues lâchement tout autour du vélum, la mère de famille s'apprête à planter le premier piquet d'angle de la tente en creusant un trou de quelques dizaines de centimètres de profondeur dans le sable, de sa main droite. Il ne s'agit pas de n'importe lequel des huit piquets qui entourent la tente: c'est le piquet avant-droit, celui de l'angle gible-sâhel (direction qui correspond au nord-ouest ou au sud-ouest selon les régions) près duquel les djinns vont être relégués une fois la tente montée et autour duquel on jettera les eaux usées et autres liquides impurs de la tente. Préalablement à cette opération, la «maîtresse de tente » prononce des paroles pieuses, le plus souvent le Bismillah (litt. «Au nom de Dieu », formule inaugurale du rituel islamique) mais aussi la fâtiḥa à laquelle on ajoutera éventuellement un autre chapitre coranique, le tout destiné à se concilier les « gens du vide» et à les maintenir à l'écart de la tente. Les autres trous ne feront pas l'objet du même rituel et pourront être réalisés par des parentes de la propriétaire de la tente venues l'aider.

Une fois la tente dressée et le mobilier installé, il n'est pas rare, dans les familles religieuses en particulier, d'inscrire sur le sable, aux quatre

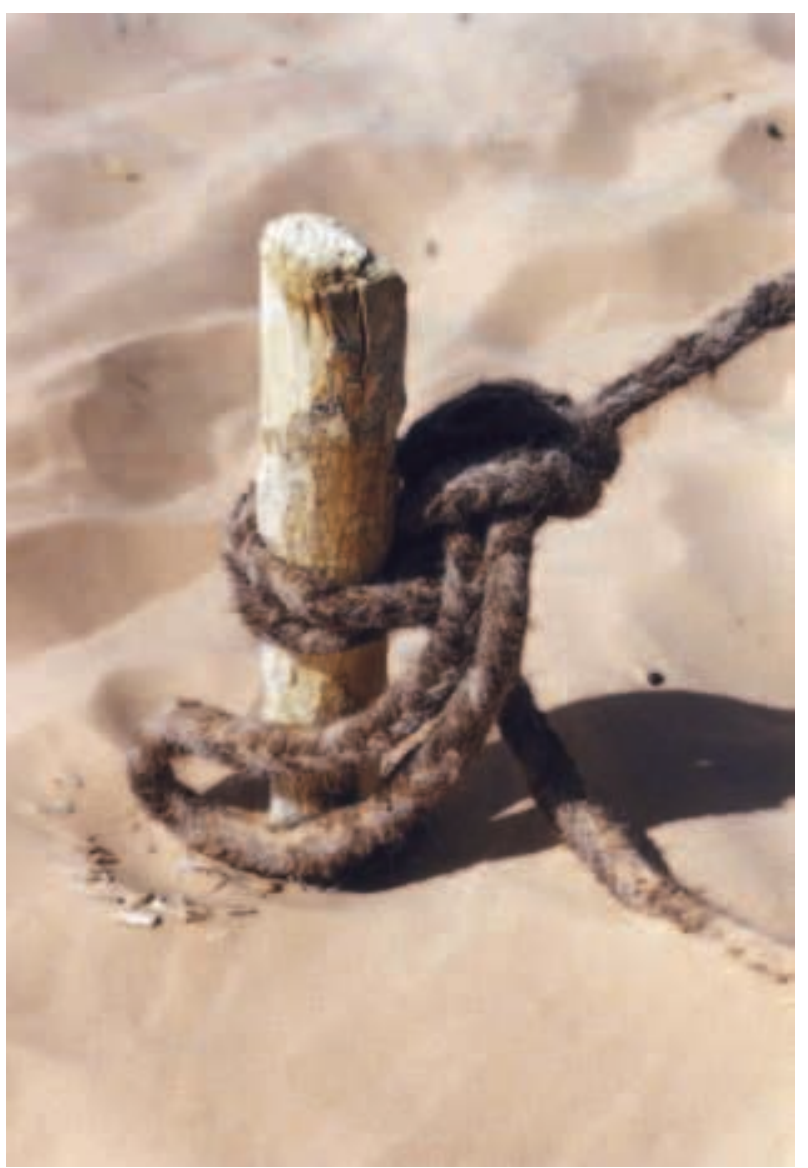

Piquet de tension d'une tente avec corde en poil de dromadaire 
coins de la tente ou devant l'entrée de celle-ci, des formules pieuses ou simplement le nom du prophète de l'Islam, puis de réciter une sourate coranique, pour protéger ses habitants. On retrouvera ces mêmes inscriptions dans le sable au moment de quitter un camp devenu insalubre et inconfortable (dâr dêrse), lorsque l'on restituera le lieu à l'immensité et aux djinns.

Ces observations montrent à quel point le contact physique avec le sable (empoigner, creuser, planter, crachoter, dessiner...) est omniprésent dans l'appropriation ritualisée de ces nouveaux espaces de vie et dans la fondation de la cellule domestique, recommencée après chaque déplacement. Mais cette appropriation ne fait sens que lorsque l'on peut s'installer dans ce nouvel espace et, pour ce faire, déployer une natte ou une couverture sur le sol. Malgré la «propreté »/ pureté du sable, il est en effet impensable de s'installer sous la tente sans avoir étendu au préalable un élément de ce que les Maures appellent le vrâš: ce terme arabe générique désigne tout ce que l'on déploie sur le sol pour le confort des occupants de l'habitation (tente ou maison). Ces éléments couvrent la quasi-totalité de l'espace intérieur de la tente: il y a généralement une grande natte ou deux, autour desquelles on va ajouter des nattes plus petites pour couvrir l'ensemble de la surface de vie. Sur ces nattes de sol, on va, à l'occasion, étendre des couvertures, spécialement lors de l'accueil d'un hôte, pour améliorer le confort au sol. Autour de ces nattes, dans les parties de la tente où l'on ne peut se tenir debout, le sol n'est pas recouvert et on laisse le sable apparent.

Les nattes sont de différents types en Mauritanie ${ }^{6}$. Les plus courantes sont « tissées» (menzûz) et présentent une trame végétale et des fils de cuir en guise de chaîne. La trame est soit à base de tiges de graminées, soit à base de nervures de feuilles de palmiers, dans les régions oasiennes du nord et de l'est de la Mauritanie. Un autre type de nattes (ḥseyr-t azarân) est caractéristique de régions sahariennes: elles mesurent généralement moins d'un mètre de large et sont presque de la même longueur que les bandes de la tente qui les abrite; la chaîne est constituée de fils en poil de dromadaire et la trame de tiges d'alfa, que l'on trouve en grande quantité dans ces régions. Enfin, de plus en plus souvent, les tentes sont dotées de nattes en brins de plastique de différentes couleurs. Elles sont commercialisées en rouleaux dans les villes importantes et sont beaucoup moins chères que les nattes traditionnelles (qui nécessitent plusieurs mois de travaux collectifs), moins prestigieuses aussi.

On notera donc la préférence accordée à la matière tissée pour affronter physiquement et symboliquement le sable ambiant, par enveloppement des habitants de la tente. La souplesse de la natte permet en effet de bénéficier de la plasticité du sable. Le textile, qu'il soit en laine et poil (vélum), en fibre végétale ou en matière plastique (nattes de sol), permet également une certaine porosité entre les matières en contact: l'air traverse le vélum de la tente, mais pas les grains de sable; les liquides renversés sur les nattes de sol traversent celles-ci et sont absorbés par le sable. Les fibres animales ou végétales sont par ailleurs considérées comme "pures » (Benfoughal 2012 : 5280) et donc particulièrement indiquées pour se prémunir du contact direct avec le sol et des puissances telluriques qu'il abrite.

Le choix et la formation de l'espace domestique apparaissent ainsi comme une recherche du meilleur compromis entre les qualités prêtées au sable, en relation avec une certaine idée du confort, et les représentations de l'espace, faste et humanisé d'un côté, stérile et dangereux de l'autre. On retrouve cette recherche d'équilibre dans les «techniques du corps » sous la tente. 


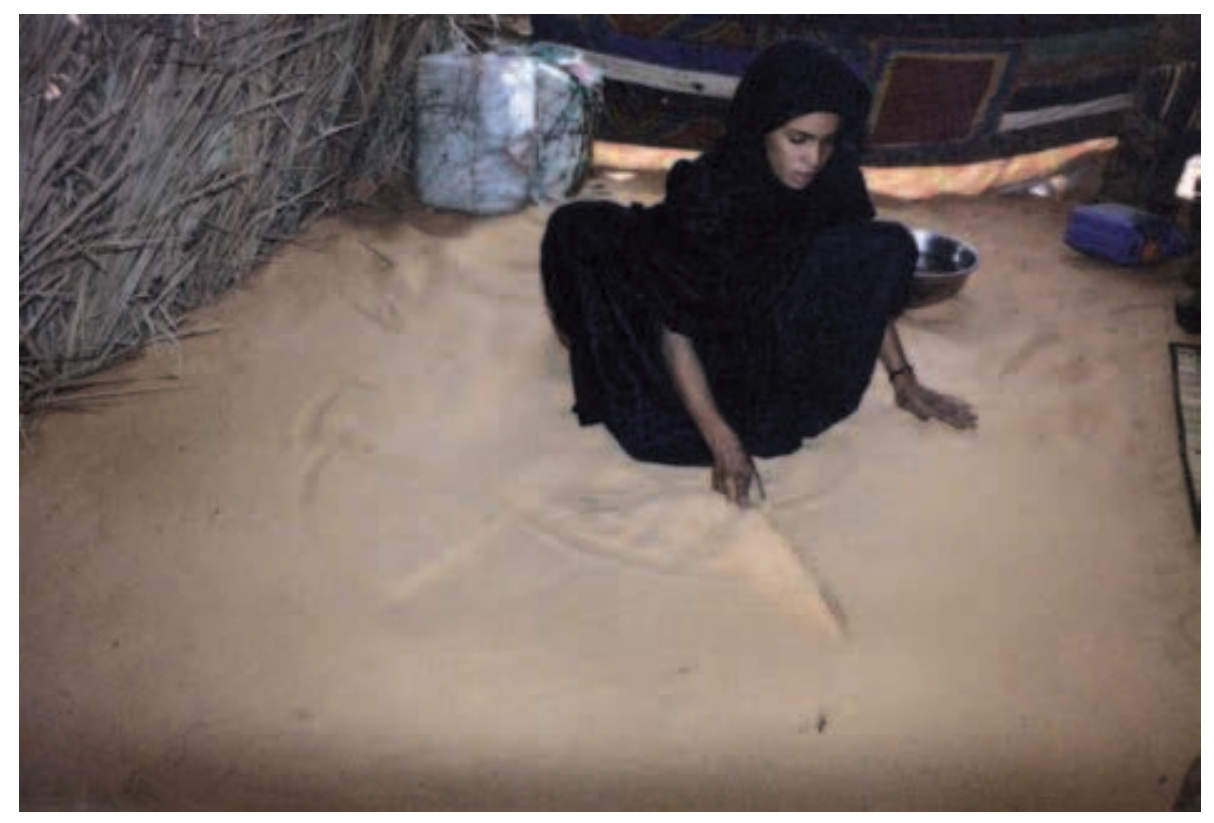

\section{Interfaces entre sable, corps et objets sous la tente}

Si la natte de sol joue effectivement un rôle central dans la médiation avec le sable, son déploiement n'en nécessite pas moins un travail préalable d'apprêt ou de préparation du sol consistant à damer le sable afin d'obtenir une surface relativement plane. Cette opération est effectuée régulièrement au cours du séjour par la mère de famille, à l'aide d'un morceau de bois (qui peut être façonné à dessein), afin de renouveler l'espace de vie ${ }^{7}$. Pour cela, les nattes sont roulées les unes après les autres, on fait passer les petits agglomérats de sable durci par des éléments liquides dans un grand tamis (habituellement utilisé pour le traitement des farines) pour les faire revenir à leur état granuleux, et on jette les petits déchets qui restent dans le tamis loin de la tente. Le sable souillé (par du sang ou de l'urine par exemple), est transporté à l'extérieur de la tente. Une fois effectuées ces opérations de tamisage et de damage du sable, on redéploie les nattes à la surface du sol.

L'espace à maintenir « propre » en priorité est celui recouvert par le vrâš (nattes de sol), qui délimite l'espace de vie. Ensuite, à un moindre degré, les femmes veillent au nettoyage du pourtour du vrâš, où l'on entrepose objets et mobilier de tente et où les individus ne s'installent jamais ${ }^{8}$, à l'exception de l'avant ou de l'arrière de la tente qui peut être occupé à certaines heures de la journée: les nattes circulent dans et autour de la tente selon les conditions climatiques et le déroulement de la vie sociale suit le « déplacement » de ces nattes. Enfin, la maîtresse de tente doit veiller à la propreté de l'espace situé à l'avant de la tente (geddâm el-hayma), espace d'accueil par lequel l'hôte va transiter, mais aussi espace que l'on occupera très souvent le soir, la nuit et au petit matin.

Les éléments considérés comme des «saletés » (usah) sous la tente sont tout d'abord la poussière ( $\dot{g} a b r a$ ) que le vent fréquent ${ }^{9}$ n'a de cesse d'apporter et qui se dépose sur les 


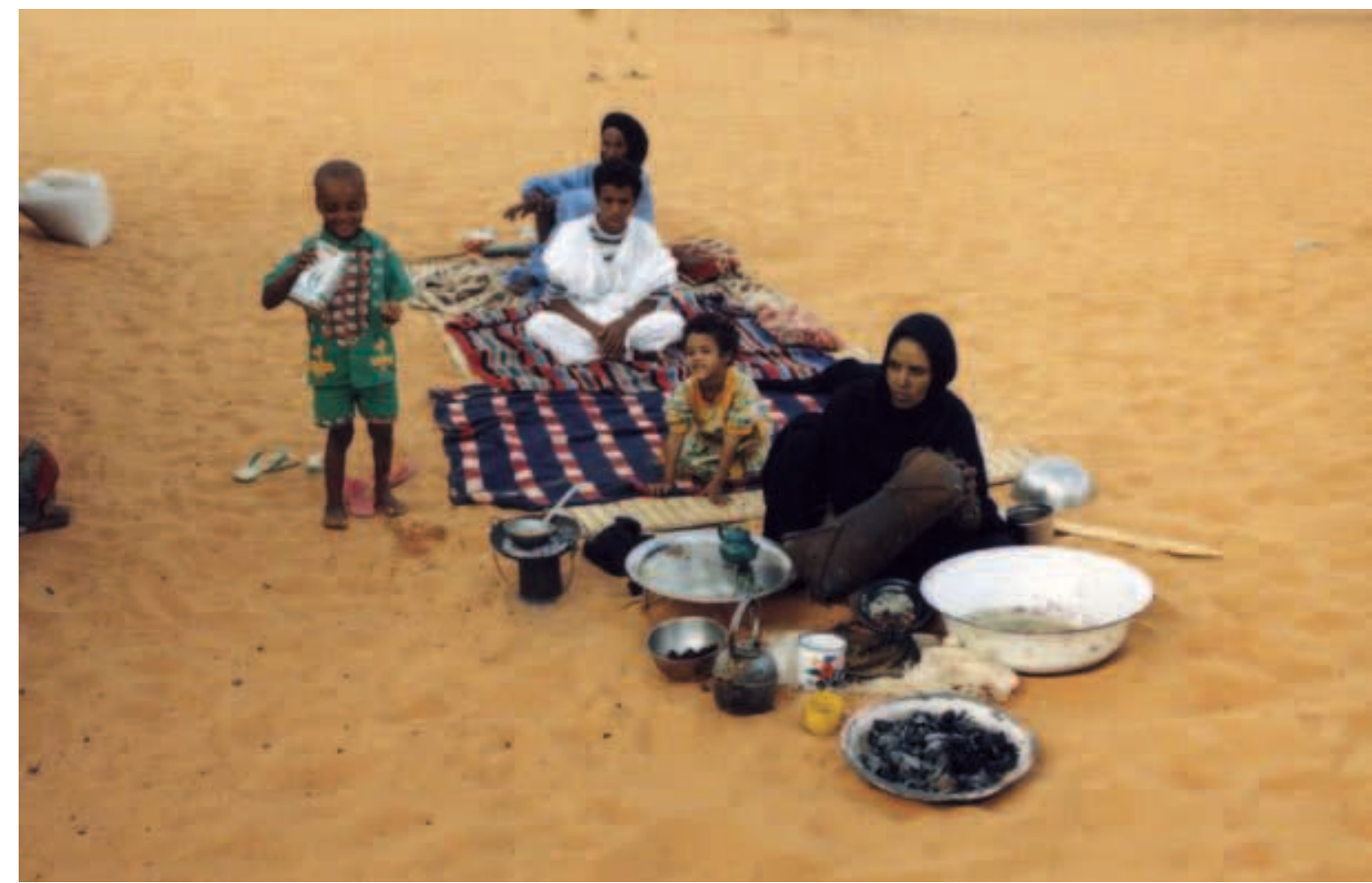

\section{Nattes installées à l'extérieur} de la tente en saison chaude nattes et les effets de la famille. Ce sont aussi les déjections des jeunes cabris et agneaux que l'on garde sous la tente ou à la périphérie de celle-ci, car trop jeunes pour partir avec le troupeau, les brindilles, les crottes de dromadaires roulées jusqu'à la tente par les scarabées. Ces éléments sont balayés hors du vrâš, à différents moments de la journée, au moyen d'un faisceau de fibres végétales rigides, ou bien simplement d'un vieux morceau de tissu. Ensuite, les détritus sont rassemblés à la main dans un vieux plat et jetés loin de la tente: ni au-devant de la tente, car il serait de mauvais goût de souiller l'horizon de ses occupants, ni à l'arrière, direction de provenance du vent dominant qui ramènerait les déchets vers l'habitation, mais plutôt au šarg (partie droite de la tente lorsqu'on lui fait face), direction opposée à celle des djinns, qui risqueraient de s'emparer de ces impuretés et de menacer l'intégrité des habitants de la tente.

Le vrâš est bien le référent à partir duquel est pensée cette géographie du propre et du sale sous la tente, qui s'inscrit dans le système cosmologique duel évoqué plus haut. Le pourtour sableux qui se trouve à l'arrière du vrâš, dans la partie sombre et intime de la tente, est ainsi le principal endroit où l'on expulse ses humeurs buccales et nasales, que l'on recouvre immédiatement de sable sec. De manière générale, il est mal vu de laisser tomber sur le sol de l'eau souillée, sous peine d'attirer les « gens du vide » à l'intérieur de l'habitation. Toute marque de liquide sur le sol apparaît en fait comme une souillure de l'espace abrité et tout agglomérat de sable causé par un liquide est aussitôt recouvert de sable sec. Il va de soi que l'égorgement rituel des petits ruminants et l'effusion de sang qu'il implique se déroulent toujours à l'extérieur et à l'écart de la tente, le sang étant considéré comme particulièrement prisé des « gens du vide »: une fois la jugulaire tranchée, 
on laisse le sang de l'animal se répandre dans le sable, puis la carcasse est déposée sur un lit de branchages pour être dépecée et débitée, et ceci avant tout pour préserver les morceaux de viande d'un contact direct avec le sable.

De même qu'on lave l'intérieur de la tente et que l'on nettoie les ustensiles de vaisselle avec du sable sec et liquide, on purifie les parties apparentes de son corps avec du sable, en vue de la prière, les « ablutions sèches » (tadammun en ar. cl., teyemmam en hass.) étant autorisées par le droit musulman pour des personnes vivant dans des environnements où les ressources hydriques sont rares, en particulier les populations du désert. Ce principe est encore très suivi en contexte bédouin en Mauritanie et s'était même maintenu lors du passage à la sédentarité et à la vie urbaine (décennies 1970, 1980 et 1990), le sable étant alors souvent matérialisé et représenté dans la maison par une pierre noire que le croyant doit toucher avant et éventuellement pendant la prière ${ }^{10}$.

Si le sable purifie, lave les objets souillés et les corps, il peut aussi être synonyme de gêne, lors de vents de sable notamment, qui sévissent pendant plusieurs jours. La technique consistant à écarter les bases des mâts de la tente pour rapprocher le vélum du sol et laisser ainsi moins de prise au vent, ne suffit généralement pas à empêcher le sable de s'introduire et de se soulever sous la tente, puis de s'inviter sous les vêtements de ses occupants et de s'immiscer dans les moindres replis de la peau. Dans ces moments, l'enveloppement dans un tissu (long voile de cotonnade pour les femmes, boubou ample et turban pour les hommes) reste le rempart le plus efficace

Tandis que l'étranger se trouve rapidement décontenancé par un vent de sable, éprouvant assez vite une sensation d'étouffement et dépensant des efforts prodigieux pour se
Carcasse en cours de débitage, déposée à l'abri du sable sur un lit de branchages

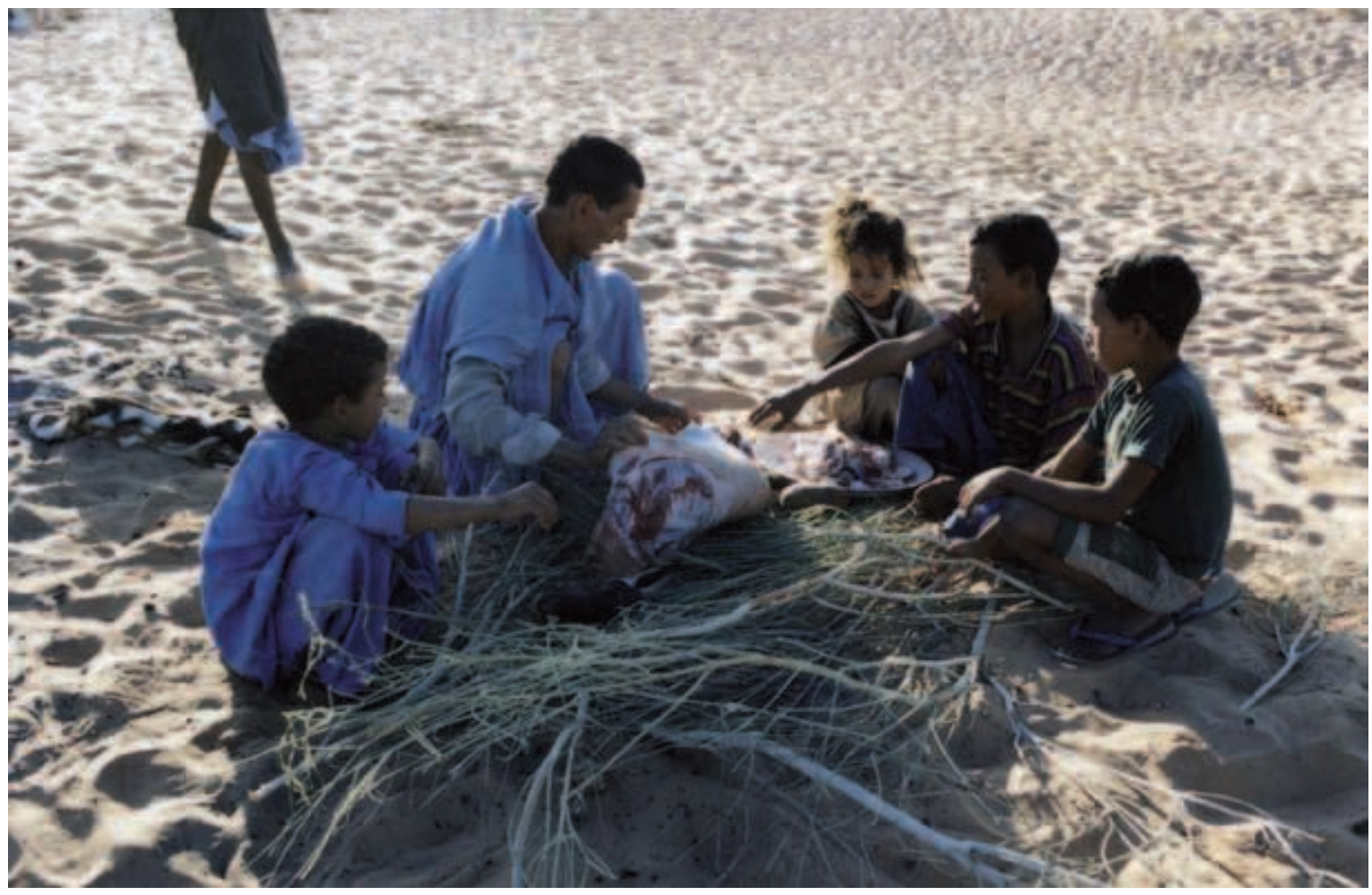




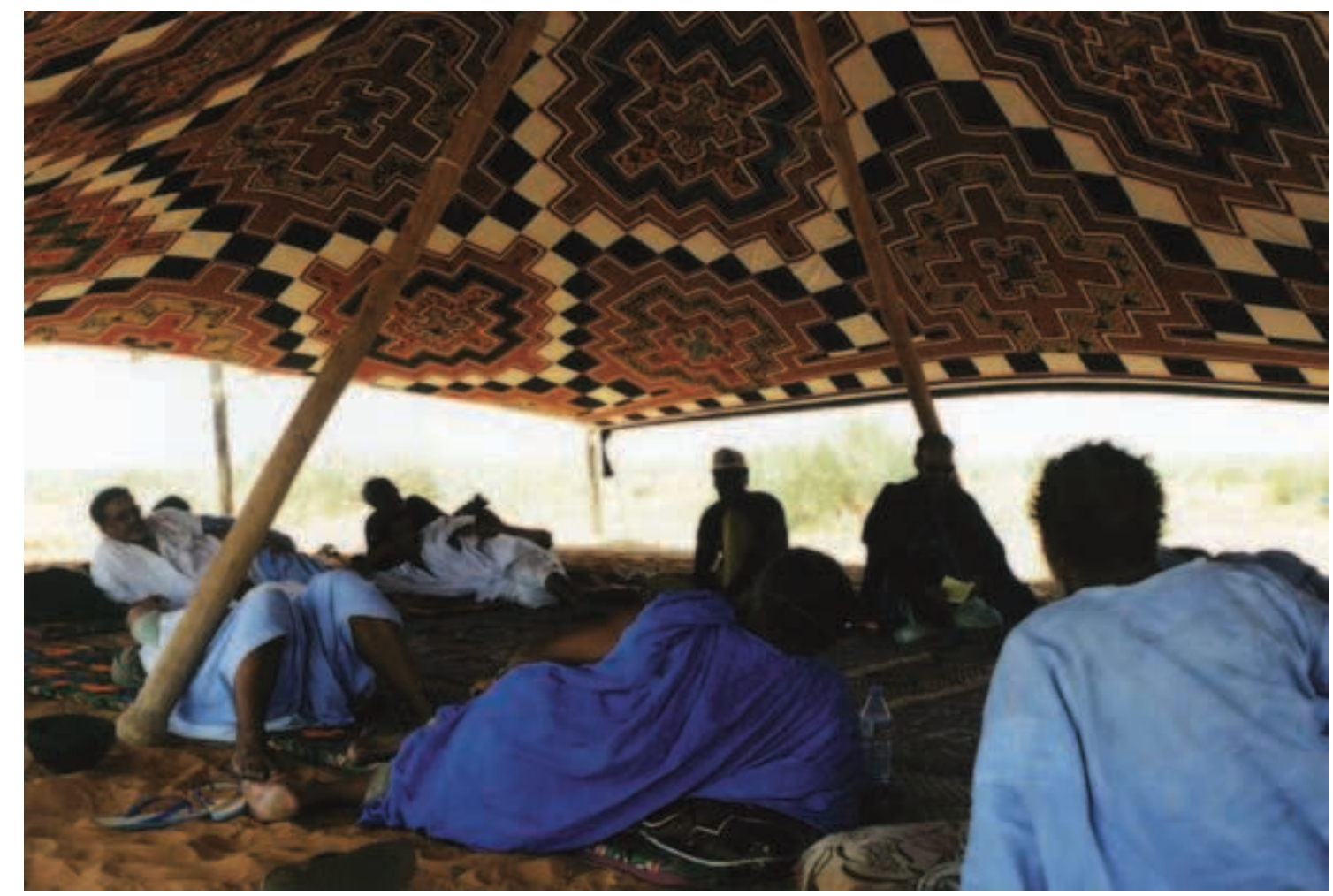

\section{Postures et occupation de l'espace}

sous la tente protéger du sable, les enfants du désert mauritanien intègrent, pour leur part, dès leur plus jeune âge, cette matière à leur schéma corporel. L'avant de la tente et sa périphérie très proche constituent en effet un terrain de jeu naturel pour eux, où ils apprennent à se mouvoir, à dessiner dans le sable, à façonner cette matière, où ils découvrent leur corps au contact du sable. La simple nécessité d'avoir à parcourir quelque distance à pied dans le sable exige une technique particulière pour ne pas se disperser dans l'effort et atteindre à une certaine "efficacité », technique qui consiste à faire de courtes foulées afin de ne pas laisser le temps au pied de s'enfoncer trop profondément dans le sol. « Faire avec » cette matière suppose, outre patience et sang froid, l'acquisition au cours de l'enfance d'un certain nombre d'aptitudes, de précautions et de gestes visant à s'adapter au mieux à ses propriétés, à en intégrer la dynamique, à en limiter les contraintes et désagréments. Bref, cela consiste à « co-(n)naître » cette matière, à acquérir un bagage mental confondant schèmes inconscients (déterminant la perception des qualités du sable), savoir-faire et représentations (Lemonnier 2000).

Ces connaissances sont ensuite mobilisées dans la recherche du maintien le plus efficace des corps, animés et inanimés, dans l'espace. D'une part, ce savoir-faire est nécessaire à un savoir être sous la tente, consistant à prendre les « bonnes » postures selon les situations sociales. Celles-ci passent notamment par une longue habitude de certaines positions du corps (assis « en tailleur», allongé sur le côté, accroupi, etc.) et par le bon usage des coussins de cuir ou de tissu qui sont à disposition et qui servent à caler les membres du corps selon les positions adoptées - la position la plus indiquée 
aux longues palabres sous la tente étant la position « tmarvîg », celle d'une personne allongée sur le flanc et accoudée à un coussin.

D’autre part, le sable permet de donner un certain maintien aux objets fabriqués, comme les parois de la tente que l'on fixe en y déversant du sable, ou en cours de fabrication, comme dans ces deux exemples de réalisation d'une vannerie en spiralé cousu ${ }^{11}$ et de fabrication d'un bât de palanquin en bois. Dans ces deux cas, le sable tient lieu de surface de travail et de support de l'action technique, tout autant qu'il conditionne la posture de la vannière et de l'artisan. Le sable enfin n'est pas incompatible avec des activités textiles de filage ou de couture de bandes de tente, la laine ou le tissu pouvant facilement être débarrassés de l'intrusion de cette matière.

Au-delà du simple support de l'action technique, le sable est encore utilisé comme matériau de traitement d'objets ou de matières. Il peut tout d'abord être utilisé, associé à d'autres ingrédients, dans la coloration de certaines matières ou objets. Il peut aussi être employé pour cuire ou faire sécher des aliments: si la cuisson au sable (de la galette de farine ou de la viande), consistant à ensevelir un aliment cru dans une cavité chauffée à l'aide de braises, est très répandue chez les Sahariens et assez bien connue ${ }^{12}$, la simple immersion pendant vingt-quatre heures de morceaux de lait caillé dans le sable (technique dite «el-gâras ») pour leur conservation, ou celle de la poutargue (gonades de mulets jaunes) chez les pêcheurs imrâgan du littoral atlantique (Boulay 2013) est plus confidentielle.

Ainsi la tente apparait-elle comme un laboratoire, certes exigu ${ }^{13}$, d'apprentissage de cette matière et de mise au point de comportements moteurs adaptés, enchâssés dans une certaine représentation du monde, comportements que l'on va reproduire après chaque déménagement, et qui s'inscrivent dans la recherche permanente du juste équilibre entre corps, matière et objets, équilibre qui a partie liée avec la maîtrise de soi et la bienséance dans cette société et, plus largement, avec l'honneur et la respectabilité. Qu'en est-il des objets présents sous la tente?

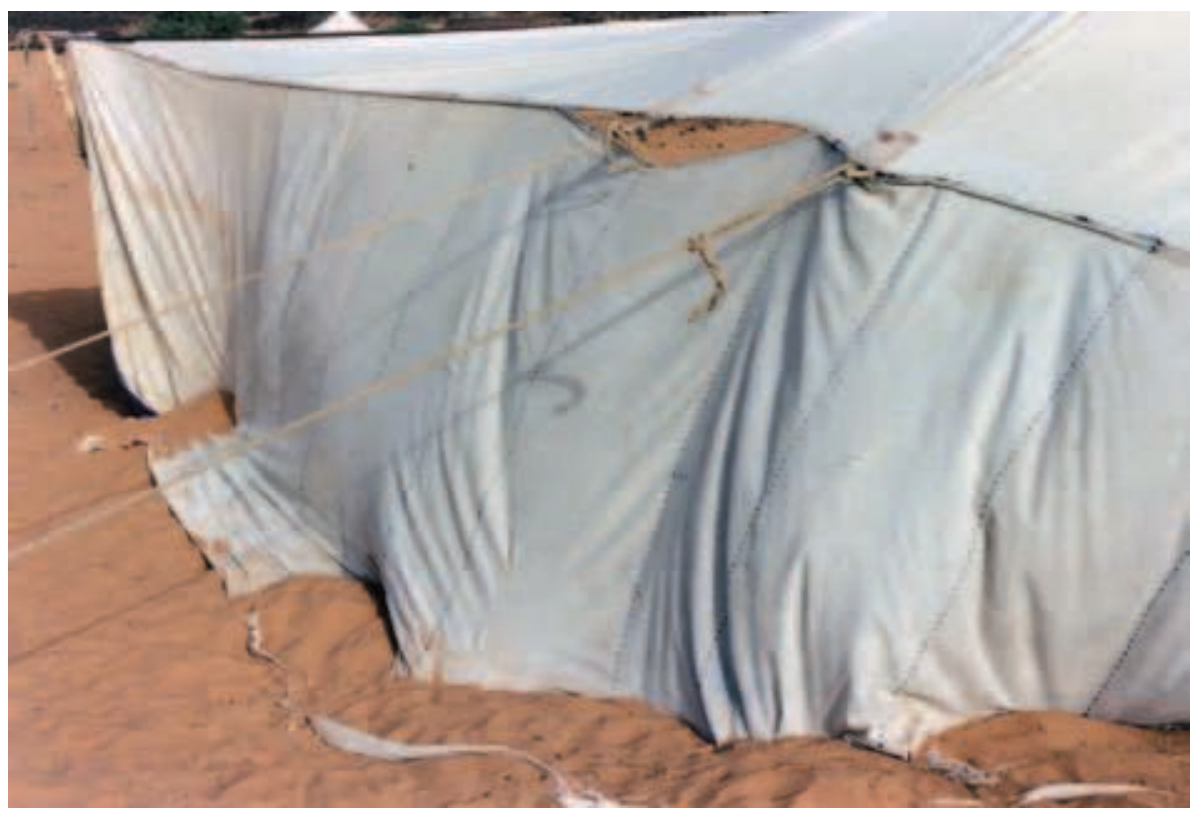




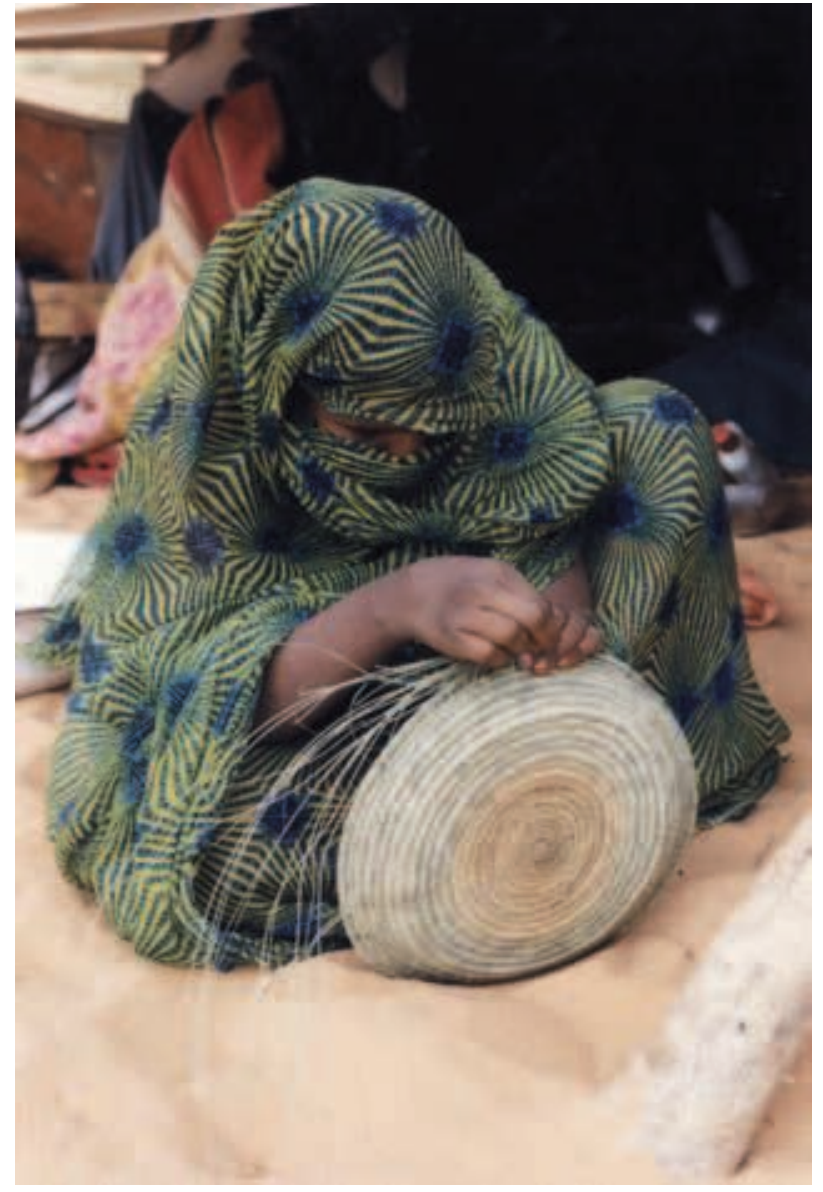

Jeune fille réalisant sur le sable une vannerie en spiralé cousu

\section{Des objets domestiques profilés?}

En admettant avec André Leroi-Gourhan que les objets sont l'ultime degré du fait (technique) et le résultat de la « rencontre de la tendance et des mille coïncidences du milieu » (LeroiGourhan 1992 : 27), on peut se demander si le sable et, plus largement, le milieu sableux a des répercussions directes sur la culture matérielle des pasteurs mauritaniens et s'ils lui confèrent un «style» (Martinelli 2005) particulier. Autrement dit, les objets de la tente ont-ils des caractéristiques qui n’auraient pas vu le jour dans un autre milieu saharien, rocheux ou rocailleux, ou dans des zones plus terreuses du Sahel? Si cette culture matérielle n'est pas adaptée à des milieux non-sableux, alors il nous faut dégager ce qui, dans la forme des objets, leur gabarit, leur nature (matériaux utilisés dans leur fabrication), mais aussi leur usage, signale une adaptation au sable, et si leur remplacement récent par des objets et des matières importés pose ou non problème à leurs utilisateurs. Cet examen oblige à isoler le déterminisme du facteur sable de celui d'autres facteurs comme l'exigence de mobilité, qui suppose des objets légers, faciles à arrimer et résistants.

On a déjà vu le rôle des objets tissés, et en particulier des nattes et des parois de tente, dans l'interface avec le sable. Ladéquation des nattes au terrain sableux ne peut être comprise que si l'on observe ce qui se passe dans d'autres « milieux ». En zone sahélienne, les pasteurs maures ne déroulent pas leurs nattes à même le sol. Ils ont recours à des nattes en bambous, surélevées par des estrades (habta muragba). Sur des sols durs voire bétonnés, en milieu urbain par exemple, on va éventuellement remplacer les nattes de sol, devenues inconfortables, par de la moquette d'importation, sur laquelle on va installer des matelas en mousse, pour atténuer la dureté du sol. Par conséquent, l'utilisation de la natte, agrémentée de coussins qui vont permettre de s'accouder et de s'allonger confortablement, ne semble véritablement optimale que sur un sol sableux et souple.

Plus évidente est la forme particulière donnée par les artisans à certains objets ou éléments architecturaux que l'on fait reposer sur le sable. Les deux mâts de la tente (rkâyz), qui soulèvent le vélum en son centre, ont tous deux des bases renflées pour éviter leur enfoncement dans le sable, ce qu'un sol d'une tout autre nature n'exigerait point. Ces mâts, confectionnés dans du bois de fromager ( djewa »), arbre qui présente le double avantage de légèreté et de robustesse, étaient généralisés à l'ensemble des familles bédouines maures, jusqu'à la raréfaction de cette essence et son remplacement par des madriers en bois d'importation. Il en est de même des pieds à base renflée de l'unique meuble de la tente: un porte-bagages servant à déposer les effets de la famille et autres réserves alimentaires, constitué de deux traverses en bois blanc de fromager montées sur quatre pieds ouvragés et joliment sculptés. Ce meuble, qui sert de palanquin lors des déplacements, se 
retrouve dans l'ensemble des tentes maures (Boulay 2006) et participe activement de leur singularité.

L'ajustement des artefacts à la matière sable se retrouve également dans les récipients «traditionnels» de la tente, qui présentent un fond creux, concave, auquel seul le sable peut donner un équilibre. Il s'agit d'écuelles de différentes tailles, dans lesquelles sont servies boissons et bouillies. Ces écuelles en bois sont déposées sur les pourtours sableux des nattes de sol. Lorsque l'on quitte les zones dunaires du désert mauritanien pour aller vers les régions plus sahéliennes, on constate un changement de forme de ces écuelles, dont le fond s'élargit et se voit doté d'une petite base plate. Il en va ainsi des récipients à traire qui sont, chez les éleveurs coutumiers des régions dunaires et en particulier chez les éleveurs camelins, à base arrondie, bombée, tandis que les éleveurs de bovins et de petits ruminants du sud du pays utilisent des vases cylindriques, dont la base permet leur stabilité sur sol plat.

Ces objets et éléments architecturaux que nous venons d'évoquer étaient jadis conçus pour durer et pour accompagner la vie du couple marié. La tente était réalisée par les parentes de l'épouse au cours des années qui suivaient le mariage, lors desquelles la jeune femme vivait sous la hayma de ses parents puis chez ses beaux-parents. Elle était ensuite régulièrement réparée et partiellement renouvelée au cours de la vie du couple. Les nattes végétales étaient également réalisées dans le cadre de travaux collectifs féminins (twîze) et transmises à l'épouse

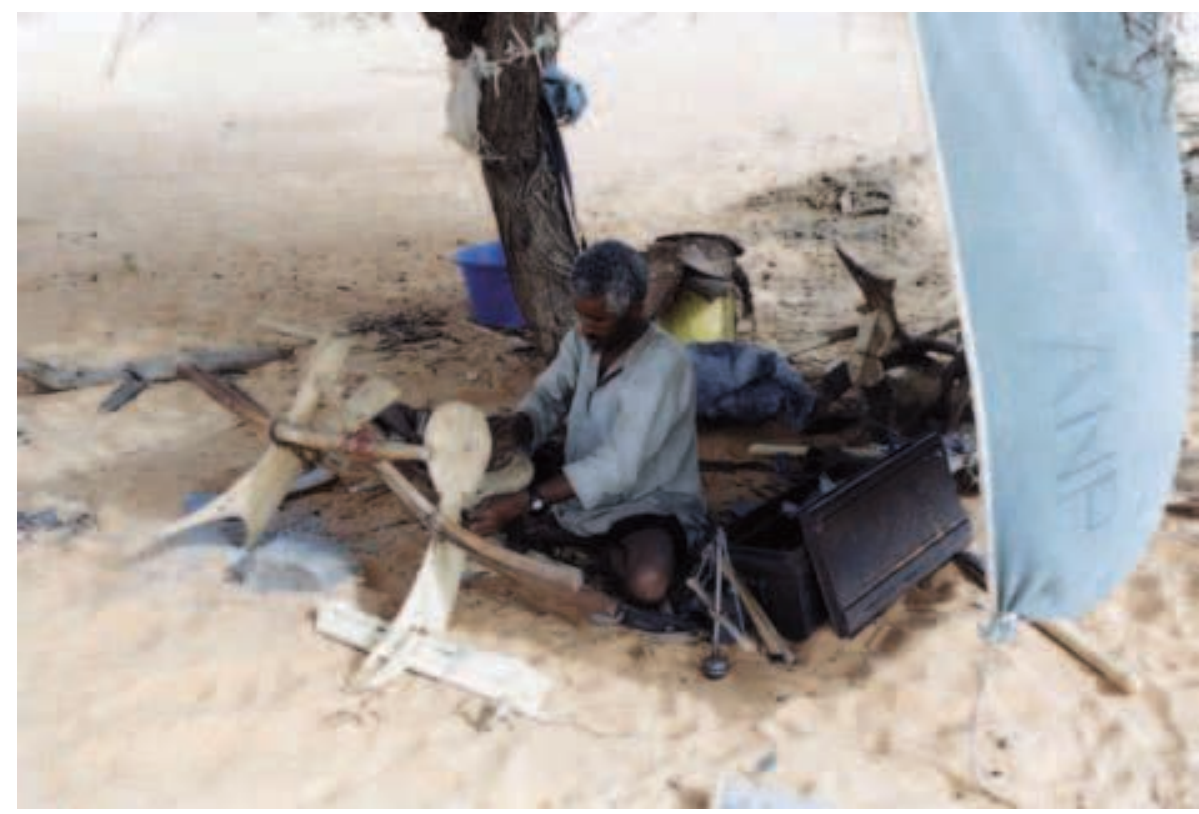

Artisan réalisant un bât de palanquin à l'ombre d'un acacia

\section{Séquence collective de couture du vélum un jour de vent}

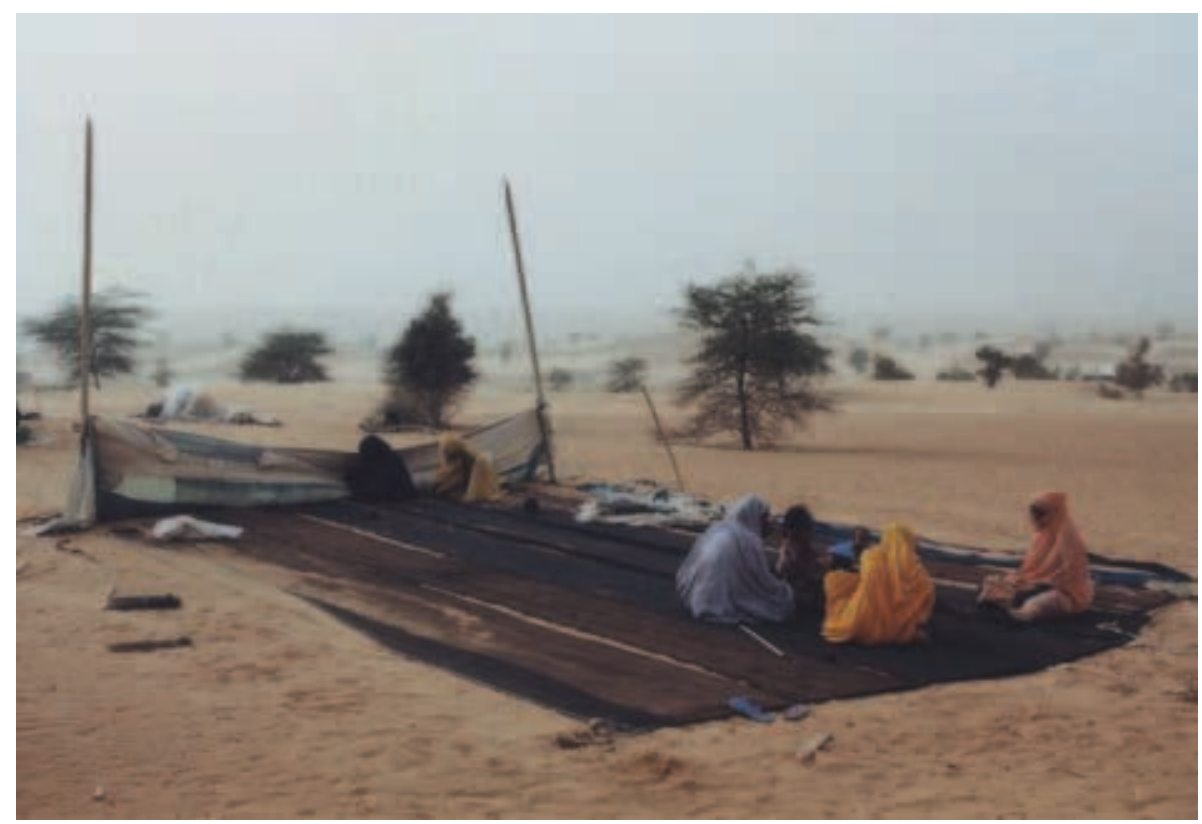



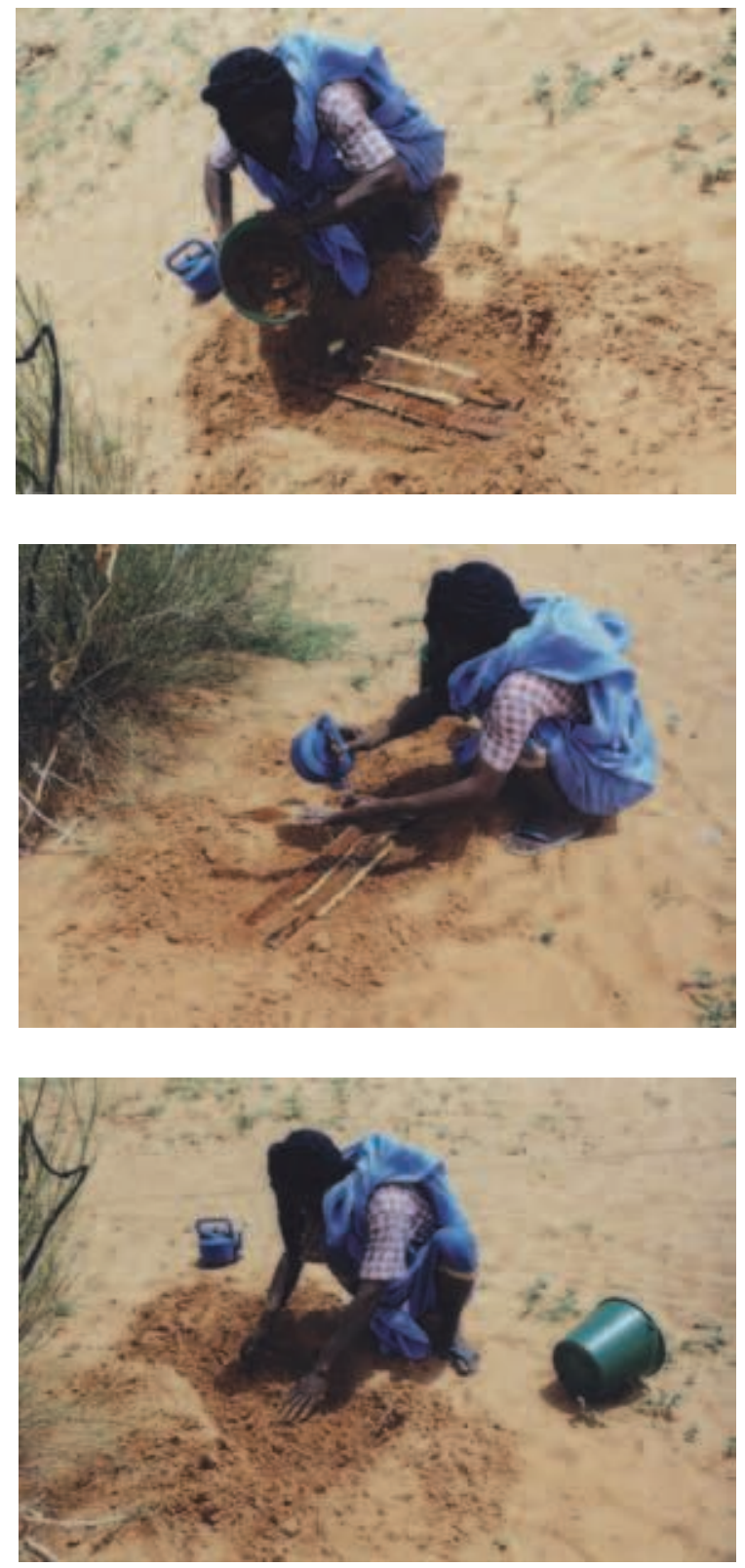

Technique de coloration de pieds de palanquin

a) après avoir été placés dans une cavité sableuse, les éléments sont abondamment mouillés, puis b) et c) recouverts de sable imbibé d'urine de chamelle. Ils sont laissés ainsi pendant deux jours et deux nuits, pour obtenir un changement de couleur spectaculaire. Le noir brillant définitif est obtenu en les enduisant de graisse ou d'huile végétale. lorsqu'elle partait vivre sous sa propre tente. Il en était de même du palanquin/porte-bagages, et des écuelles avec lesquelles elle nourrirait sa famille et ses hôtes. La réalisation et l'obtention de ces objets par l'épouse s'inscrivaient dans le cadre d'un réseau de relations sociales où circulaient matières, forces de travail et objets, sur la longue durée. Ainsi, ces objets pouvaient-ils être réparés à tout moment, en recourant ou non aux services d'un artisan spécialisé.

Aujourd'hui, l'espace domestique des Sahariens est envahi d'objets et de matières nouvelles que l'on s'approprie de différentes manières ${ }^{14}$. En Mauritanie, les objets d'importation pallient le départ massif des artisans en ville, notamment depuis les grandes périodes de sécheresse des années 1970 et 1980. Ces objets et matières d'importation, relativement bon marché, comme les cotonnades industrielles qui sont désormais utilisées dans la réalisation des vélums et parois de tentes, ou comme les nattes en plastique, présentent l'énorme avantage de faire l'économie de travaux collectifs féminins extrêmement longs et fastidieux. Il est des régions où les écuelles en métal, émaillé ou non, ont pratiquement remplacé les récipients en bois sculpté, qui restent néanmoins réservés à la boisson d'accueil des hôtes de la tente. Certains récipients en plastique, réalisés à partir d'objets de récupération comme des flotteurs de filets de pêche sur la côte atlantique du Sahara, sont des répliques exactes des écuelles «traditionnelles». Seul le palanquin/porte-bagages semble se maintenir à peu près tel quel, n'ayant pas encore d'équivalent d'importation.

Ces nouveaux objets et matières ont été très vite appropriés par les pasteurs mauritaniens et intégrés dans les circuits d'échanges d'objets entre familles, qui passent désormais par la médiation de l'argent. Ils ont même un certain succès chez les jeunes générations qui voient là un ancrage dans la modernité. Le plastique est par exemple très bien adapté, de par sa légèreté, au nomadisme, et bien accepté pour la variété des couleurs qu'il offre. Mais sa résistance semble faible et exige un renouvellement très fréquent. Les nattes en plastique, au contact permanent du sable, présentent une obsolescence bien plus rapide que les nattes végétales, qui, par ailleurs, ne nécessitent pas d'investissement financier puisque fabriquées avec des matériaux disponibles localement.

Si les matières, l'esthétique et les techniques de fabrication et de réparation peuvent changer, ces objets usuels adaptés aux milieux sableux et à la mobilité se maintiennent aussi parce qu'ils continuent de faire sens dans cette société, au-delà de leur efficacité technique. Le style que la tente et ses objets, leur forme, leur usage, leur agencement, contribuent à incarner et qui diffère par exemple assez nettement de la culture matérielle des Touaregs maliens voisins (Hincker 2005a), renvoie pour les membres de cette société à une certaine esthétique partagée de la vie et du bien-être au désert - représentations 

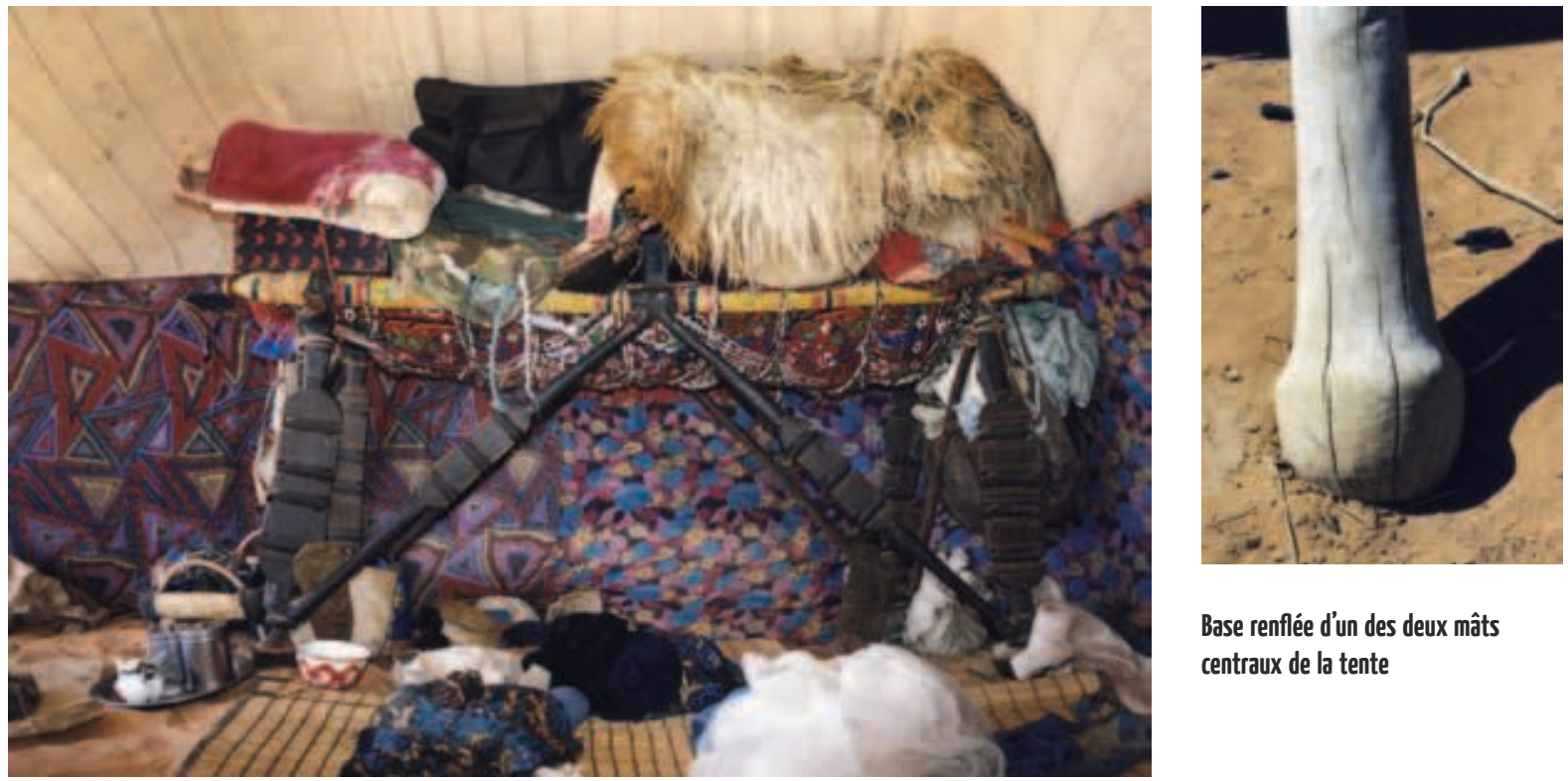

Base renflée d'un des deux mâts centraux de la tente

\section{Porte-bagages}

Seul « meuble » de la tente permettant de ranger les affaires de la famille et quelques réserves alimentaires, et servant, retourné, de palanquin.

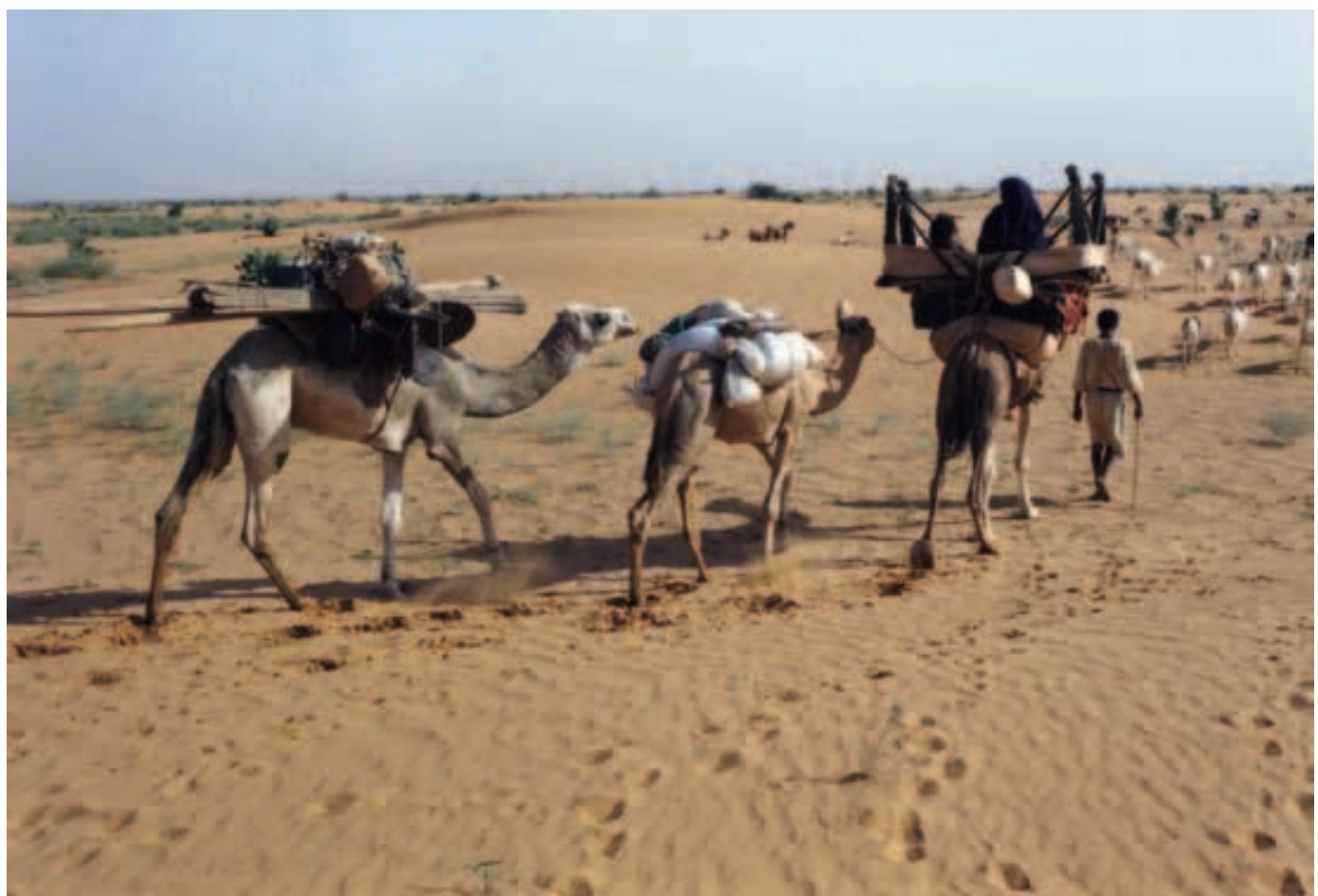

Famille en déplacement et utilisation du porte-bagages dans sa version palanquin 

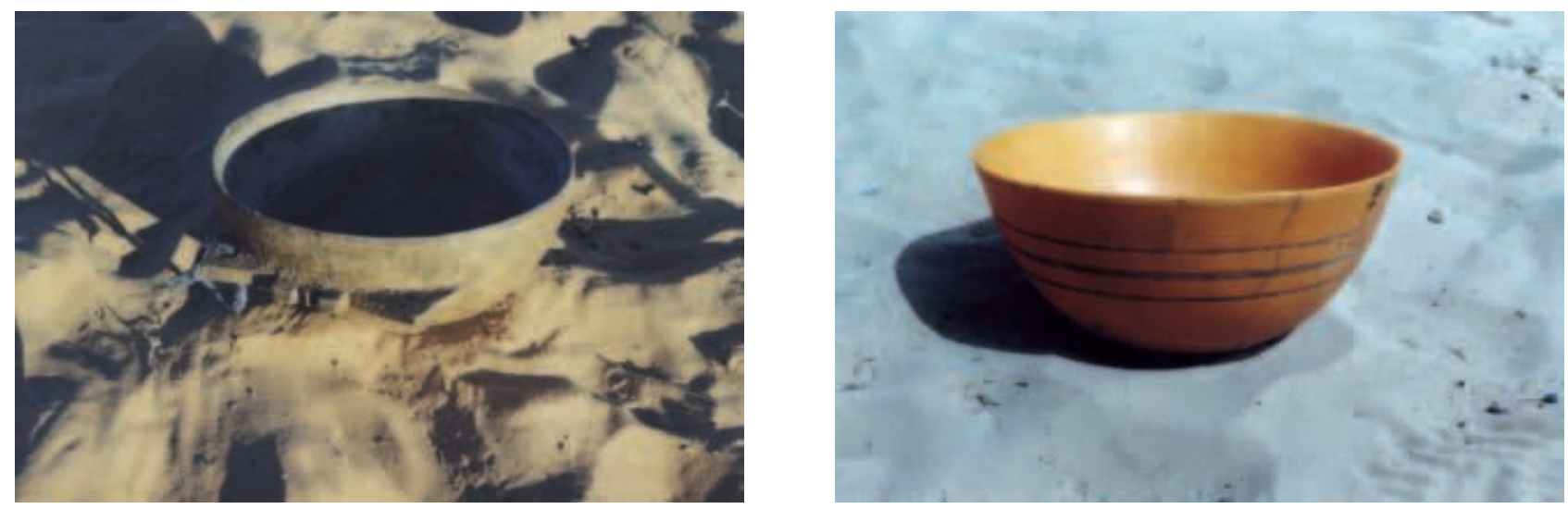

Écuelle à traire les chamelles, monoxyle, avec poignée et attache

Réplique d'écuelle traditionnelle réalisée à partir d'une bouée de filet de pêche intégrant également des paysages de dunes, du bétail gras et certains aliments emblématiques de la vie bédouine comme le lait et la viande. C'est ce style qui continue de déclencher l'émotion de celles et ceux qui se reconnaissent dans cette culture matérielle (Hincker 2005b) - et dans ces quelques objets phares que sont la natte de sol, les coussins, les écuelles de bois et le palanquin/porte-bagages -, univers matériel que l'on va tenter de recréer après chaque déplacement, ou dans des démarches collectives de retour à la vie bédouine, y compris lorsqu'elles émanent de populations récemment citadinisées.

\section{Sable, béton et bitume}

Que devient alors cet équilibre entre sujets et matière ambiante lorsque les pasteurs nomades se sédentarisent? Quelle nouvelle place est faite au sable lorsque l'on vit sur/dans du béton, entre quatre murs? Telles sont les questions par lesquelles nous conclurons cette réflexion.

À première vue, le passage de la vie sous la tente, au contact du sable, à l'habitation en dur crée une rupture avec cette matière ambiante. La vie dans un baraquement de taule, puis dans une maison d'une ou plusieurs pièces, voire dans une villa comme celles que l'on voit pousser depuis deux décennies dans les quartiers nantis de la capitale mauritanienne, Nouakchott, laisse à penser que l'on est mieux prémuni du sable, notamment lors des vents violents, fréquents à certaines saisons. La ville de Nouakchott a été largement construite, depuis qu'elle a été choisie comme capitale nationale, sur des terrains sableux puis même dunaires ces dernières années (les parties nord et est de la ville étant entourées de cordons dunaires imposants). Or le vent fait rentrer le sable partout et celui-ci devient rapidement un intrus lorsque l'on vit dans un habitat en dur et que l'on ne peut plus déménager.

Alors que le sable était valorisé en milieu bédouin pour sa capacité à faire disparaître les éléments impurs présents dans l'espace domestique, il indispose en ville et devient, à son tour, l'élément indésirable à évacuer de l'habitation. Devant les maisons ou les petites boutiques de quartier, on n'hésite pas à asperger le sol d'eau pour mieux neutraliser l'élément sableux en lui donnant, pour quelques heures seulement, une consistance solide, et pour limiter son intrusion dans l'espace de vie. À Nouakchott, si l'on « baigne » dans 
le sable, de nouvelles matières solides et totalement hermétiques au sable médiatisent et reconfigurent toutefois cette relation. Le mobilier domestique des citadins se transforme tout en restant très sommaire, mais la vie à même le sol se maintient à peu près partout : seuls les matelas épais et la moquette ont fait leur apparition dans les habitations.

Le « sable blanc » reste cependant recherché par les citadins souhaitant passer un bon moment hors de la maison, où la vie reste souvent synonyme d'enfermement. On part alors à la recherche d'une petite dune, en pleine ville comme on en trouve encore dans certains quartiers de Nouakchott ou dans sa périphérie, sur laquelle on va déployer une jolie natte et disposer quelques coussins, pour passer du bon temps autour d'un thé. Ces démarches récréatives urbaines peuvent donner lieu à une forme de transhumance estivale qui voit certaines familles partir séjourner quelques semaines dans le désert sous la tente, et retrouver cette vie bédouine qui nourrit la nostalgie de générations de citadins maures. Ces transhumances récréatives annuelles sont intéressantes à observer notamment parce que ces citadins, parfois coupés de la vie dans le désert depuis plusieurs décennies, doivent réapprendre, ou tout simplement apprendre pour les plus jeunes, à composer avec le sable, et plus généralement avec les petits inconvénients de la vie dans le désert et notamment la présence d'insectes et d'animaux indésirables. Ces moments longtemps fantasmés de retour à la vie bédouine le temps d'un week-end ou de vacances scolaires, peuvent à l'occasion déboucher sur des sensations d'inconfort, que l'on pourrait imputer, en suivant Warnier (1999) et Rosselin (2002), à une inadéquation et à un nécessaire (ré)ajustement des conduites motrices et du schéma corporel de ces citadins à la vie sur le sable et sous la tente.
Camp spontané de citadins installés le long de la route nationale Nouakchott-Rosso et repartant vivre dans le désert le temps des vacances scolaires ou d'un week-end

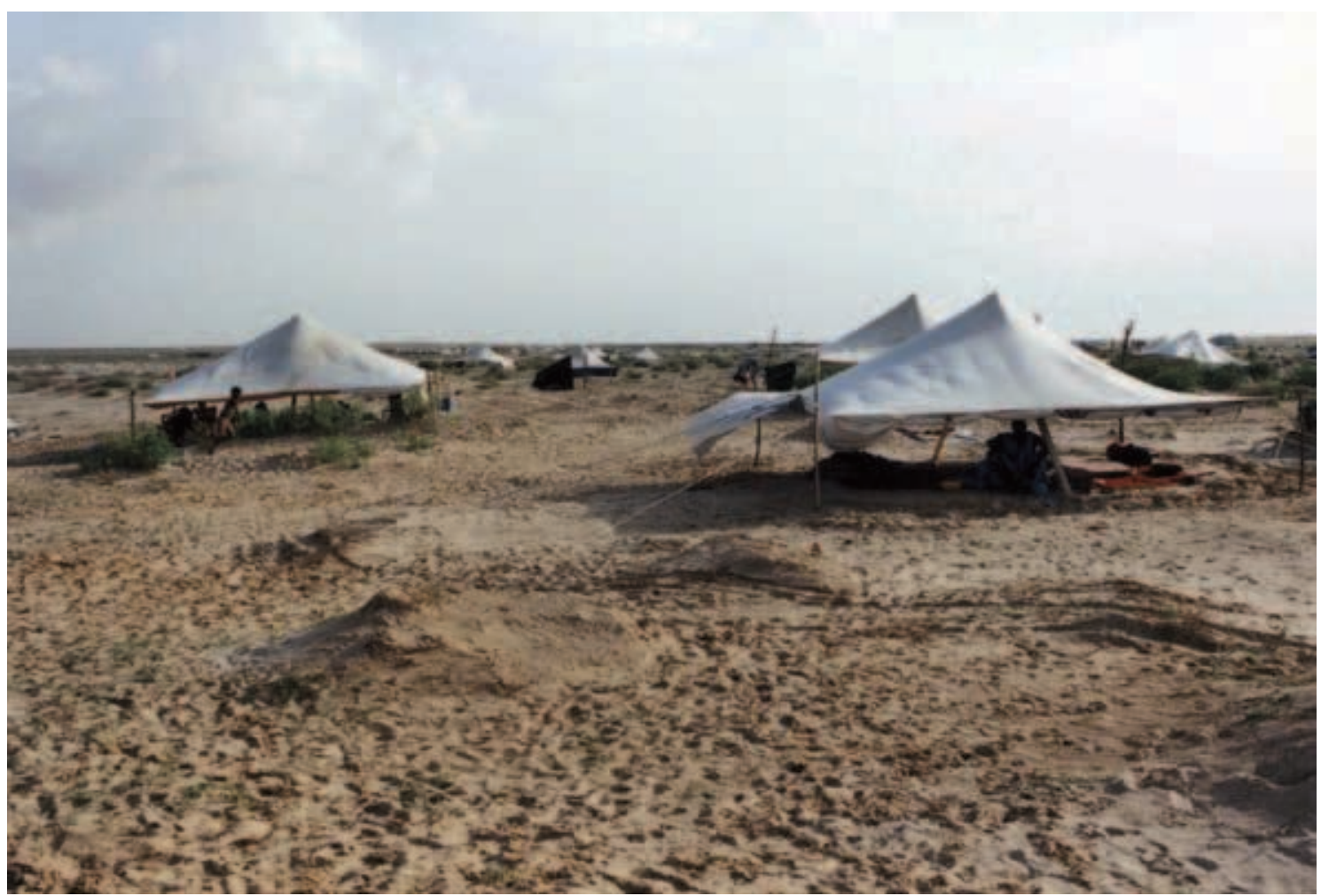




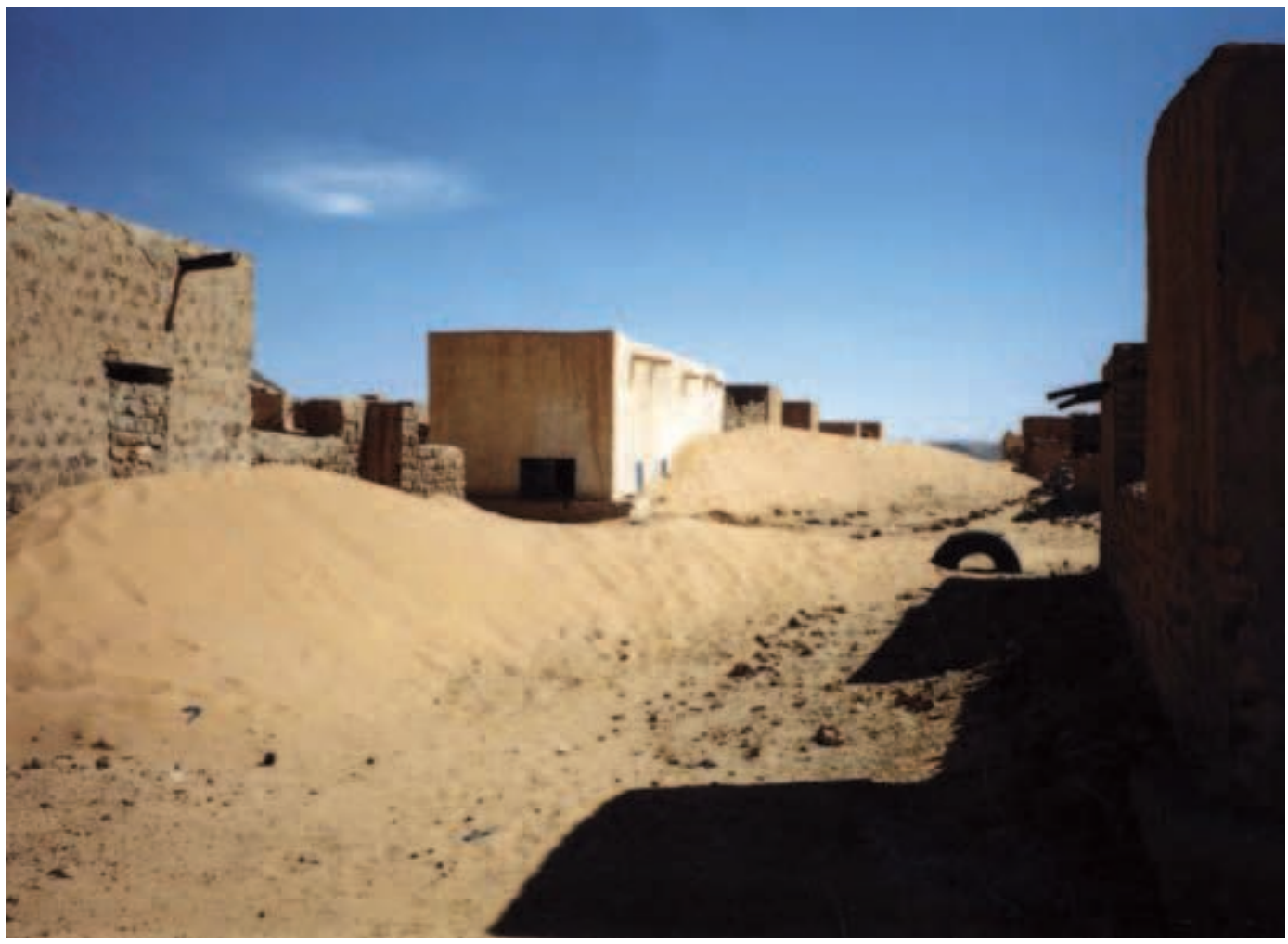

Ensablement d'une rue de Tijikja

(Tagant, Mauritanie)

On pourrait faire un constat similaire au sujet des objets du quotidien présents en ville, qui ont tendance à proliférer tout en montrant une fragilité croissante face à cette matière ambiante envahissante. Nous pensons évidemment ici aux nouvelles technologies que sont l'ordinateur ou le téléphone portable, qui semblent particulièrement vulnérables à l'intrusion ravageuse des petits grains de silice. Nous songeons également aux automobiles d'importation, à la mécanique de plus en plus sophistiquée, assistée par ordinateur, qui périclitent rapidement dans cet environnement, faisant de la capitale mauritanienne un gigantesque cimetière automobile, et ceci malgré la présence nombreuse de garages à ciel ouvert, où l'on travaille les pieds dans le sable et où l'on essaie de ranimer ces objets prématurément obsolètes. Sédentarité et prolifération de matières et d'objets importés donnent naissance à de nombreux dépôts d'ordure spontanés, que le sable n’a plus le temps de recouvrir.

Le changement de mode de vie semble donc avoir fait perdre au sable sa capacité et sa fonction sociale de (di)gestion des restes et des déchets humains. Les routes goudronnées se multiplient dans la ville, avec l'explosion du parc automobile. Béton et bitume semblent affronter le sable dans une guerre entre des matières artificielles, d'un côté, et une matière naturelle, de l'autre, sur laquelle l'homme n'a que l'illusion de gagner du terrain, au prix d'efforts prodigieux: le désensablement des rues des villes et bourgades est une histoire sans fin, de même que celui des quelques routes qui sillonnent le pays et qui doivent régulièrement être libérées de ces amoncellements de milliards de milliards... de grains de sable. 
Ces reconfigurations du rapport social au sable peuvent enfin être motivées par de nouveaux discours religieux, en provenance du Moyen-Orient, qui prônent par exemple l'abandon du teyemmam, les « ablutions au sable ». Celles-ci, généralisées en milieu nomade maure, s'étaient, comme nous l'avons signalé plus haut, largement maintenues en ville ${ }^{15}$ lors des premières décennies de sédentarisation, même si substituant parfois au sable une pierre noire, dont le toucher symbolisait le contact avec la «terre » (trâb). Aujourd'hui, en milieu urbain, ces pratiques de purification au sable font débat ${ }^{16}$ car considérées comme d'un autre âge et irrecevables par certains leaders religieux qui ne tolèrent que les ablutions à l'eau douce. Ces positions, même si encore négligées par un grand nombre de pratiquants mauritaniens, influencent néanmoins progressivement les pratiques religieuses, notamment des jeunes citadins qui n'ont connu que la vie en ville, et ce malgré la rareté et la cherté d'une autre matière sur laquelle il y aurait aussi beaucoup à réfléchir: l'eau.

\section{$\&$}

Ce dernier exemple illustre à quel point le rapport social au sable est dynamique, fruit d'un va-et-vient permanent entre, d'une part, les pressions de la matière sur les individus, leurs représentations et pratiques quotidiennes et, d'autre part, les actions des individus, plus ou moins efficaces techniquement et symboliquement, sur la matière. «Faire avec » le sable suppose donc de créer la relation la moins disharmonieuse possible avec ce milieu ambiant et passe par la production et la transmission d'un savoir être et d'un savoir-faire, qui certes participent d'une identité culturelle partagée, mais qui sont éminemment vulnérables aux moindres modifications du contexte social. 


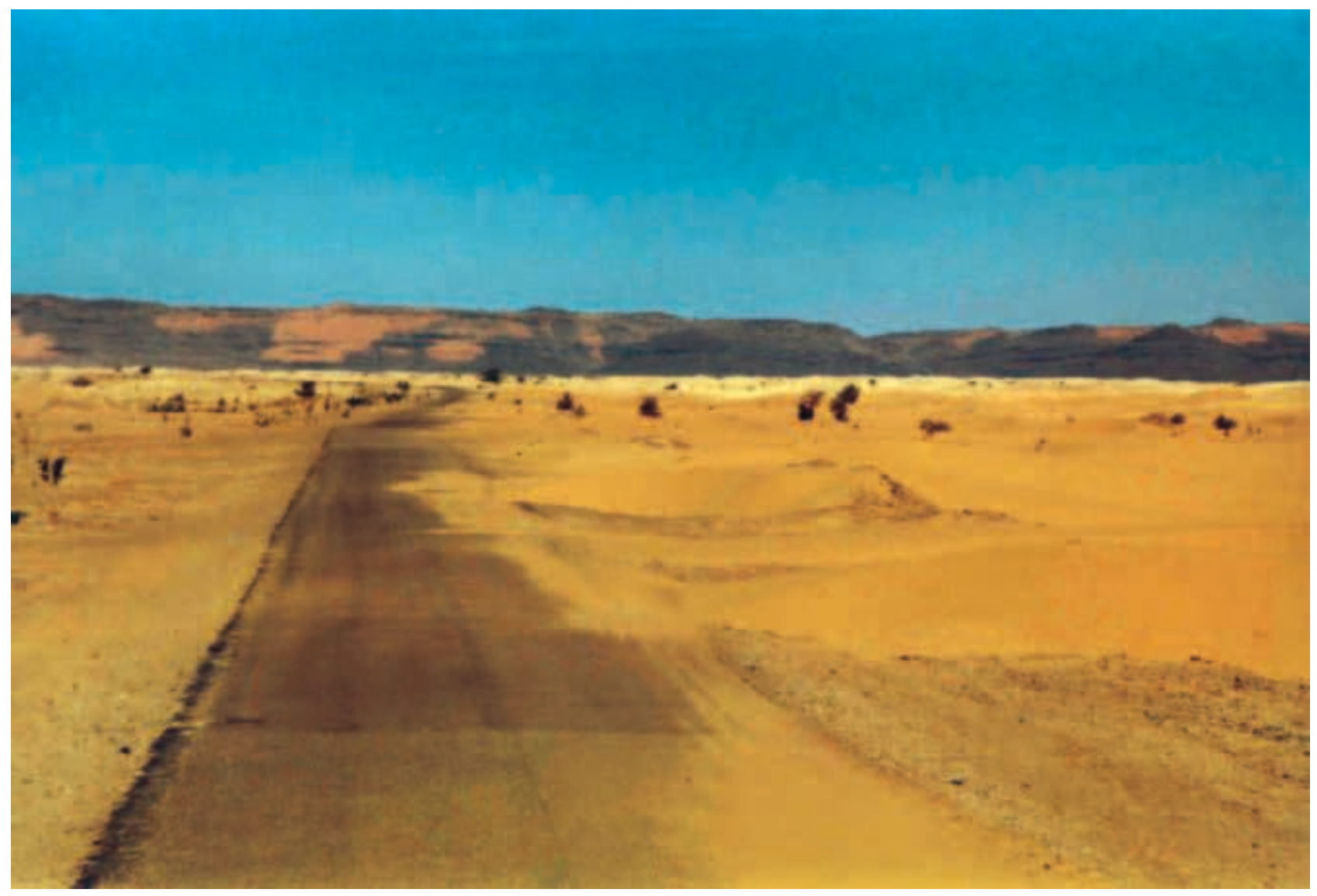

Route bitumée conduisant au pied du plateau du Tagant 


\section{NOTES}

Photo d'ouverture: Tente brune «traditionnelle » des pasteurs nomades ouest sahariens (Cl. S. Boulay).

Les illustrations de cet article sont à mettre au crédit de l'auteur, Sébastien Boulay.

* Normes de transcription du dialecte arabe mauritanien, le ḥassâniyya:

$\underline{t}$, th anglais de « think »; $h$, h aspiré; $h$, vélaire sonore, jota espagnole; $\partial$, th anglais de « the »; $\underline{d j}$, dentale sonore palatalisée; $\check{s}$, ch français; $s$, s emphatique; $d$, d emphatique; $t$, t emphatique; $z$, z emphatique; $\underline{\partial}$, $\partial$ emphatique; ${ }^{c}$, spirante sonore émise par le larynx comprimé; $\dot{g}$, r grasseyé; les voyelles longues sont indiquées par un accent circonflexe: â, û, $\hat{\imath}$.

1. Dans le premier cas, l'architecture est qualifiée de « hayma », mot utilisé dans tout le monde arabe pour désigner la tente bédouine. Dans le second cas, celui d'une structure à arceaux plus petite - présente également chez les Touaregs (Nicolaisen 1997) ou les Toubous (Prussin 1995) des pays sahariens voisins -, les Maures utilisent strictement le terme « benye » qui renvoie à une structure plus confidentielle, utilisée soit comme double toit sous la hayma, soit comme abri nocturne et discret des jeunes époux, soit enfin comme annexe de la tente utilisée dans les périodes de fréquents déplacements (Boulay 2007).

2. L'oasis et la ville, avec leurs formes propres d'habitat, offrent il est vrai aux pasteurs ouest sahariens d'autres espaces de vie sociale.

3. Voir le texte de Catherine Taine-Cheikh dans ce numéro.

4. Cette dualité entre blancheur et noirceur se retrouve dans l'ensemble du système de valeurs des Maures, et en premier lieu dans la distinction hiérarchique entre «Blancs » (Bidân), ainsi que se nomment les Maures revendiquant une noble condition, et « Noirs » (Sudân), qui désignent les Maures de statut ou d'origine servile.

5. Les pasteurs ouest sahariens se représentent leur univers, lointain et proche, dans une opposition entre le monde humanisé des hommes et des campements, conçu comme plein et faste, et le monde « vide », stérile et dangereux du désert, considéré comme le domaine des djinns appelés « gens du vide » (ahl le-hlle) ou « gens de l'immensité » $\left(\right.$ ahll l-us $\left.\hat{a}^{c}\right)$. On retrouve cette représentation duelle de l'univers notamment chez les Touaregs voisins (Casajus 1987; Claudot-Hawad 1993).

6. Sur les nattes en Afrique du Nord, on se reportera au récent article de synthèse de T. Benfoughal (2012), qui présente tout à la fois les différents types de nattes utilisés, les techniques de fabrication, l'organisation de la production et de la commercialisation, les dimensions symboliques et rituelles.
7. Une « tente propre » c'est avant tout une tente dont l'environnement intérieur, le sol en particulier, est débarrassé de toute souillure. Mais c'est aussi un espace où les objets sont à leur place, où les effets de la famille sont rangés d'une certaine manière sur le palanquin porte-bagages. L'acception du terme mașgûl dépasse par conséquent la dimension purement hygiénique pour englober celle de l'ordre matériel.

8. Ces endroits où sont placés mobilier et vivres sont souvent le refuge idéal des scorpions.

9. Surtout lors de la période qui précède la geytna (cueillette des dattes), en mai-juin, durant laquelle le vent violent de nord-est, sec et chaud, rend la vie très difficile et inconfortable.

10. Selon les personnes que nous avons interrogées sur cette pratique, toute pierre peut faire l'affaire, mais celle-ci doit être très dure et préservée de toute souillure.

11. Sur la vannerie saharienne et ses techniques, voir notamment: Benfoughal 2006.

12. Voir le texte de M.-L. Gélard dans ce numéro.

13. Voir le travail de Céline Rosselin (2002) sur les processus d'incorporation des objets dans les logements d'une pièce.

14. Voir à ce sujet les travaux de Benfoughal (2002) et de Gosselain, Zeebroek Decroly et Decroly (2009).

15. Une amie nous a ainsi dernièrement raconté avoir découvert, à la fin des années 1990, un petit tas de sable déposé sur un sac en papier dans le bureau d'un riche homme d'affaires mauritanien, vraisemblablement encore très attaché à cette pratique des «ablutions » au sable... ou au sable tout court!

16. Un religieux mauritanien a récemment défendu cette pratique du lavement au sable comme une possibilité offerte au croyant, retardé pour quelque raison, de gagner du temps en ne procédant pas aux ablutions à l'eau.

\section{POUR CITER CET ARTICLE}

Boulay, S. 2014 Habiter ou «faire avec » le sable..., in S. Boulay \& M.-L. Gélard, Vivre le sable! Corps, matière et sociétés, Techniques \& Culture 61 : 76-99. 


\section{RÉFÉRENCES}

Benfoughal, T. 2012 Nattes in Encyclopédie berbère. Paris et Aix-en-Provence: Éditions Peeters, Paris-Louvain-Walpole, XXXIII : 5260-5294.

- 2006 De la matière première à l'objet tressé: la vannerie du Sahara maghrébin in T. Benfoughal \& S. Boulay (dir.) Sahara : identités et mutations sociales en objets, Journal des Africanistes 76 (1) : 141-163.

- 2002 Ces objets qui viennent d'ailleurs in H. Claudot-Hawad (dir.) Voyager d'un point de vue nomade. Paris: IREMAM et Paris-Méditerranée: 113-135.

Boulay, S. 2013 Pêcheurs imraguen du Sahara atlantique. Mutations techniques et changements sociaux des années 1970 à nos jours. Paris: Karthala.

— 2007 The Role of Architecture in Society: Structuring of the Family Unit Among The Moorish Pastoral Nomads of Mauritania, Nomadic Peoples 11 (1): 49-67.

— $2006 \mathrm{La}$ « double vie» du porte-bagages: un objet singulier sous la tente des Maures (Mauritanie) in T. Benfoughal \& S. Boulay (dir.) Sahara: identités et mutations sociales en objets. Journal des Africanistes 76-1: 121-139.

— 2005 Genèse, représentations et usages de l'espace de la famille chez les Bédouins maures (Mauritanie) in J.-Y. Authier \& C. Bidou (dir.) La famille dans tous ses espaces. Espaces et Sociétés 120-121: 141-161.

- 2003 La Tente dans la société maure (Mauritanie). Ethnologie d’une culture matérielle bédouine en mutations. Thèse de doctorat en ethnologie du Muséum national d'Histoire naturelle, Paris.

Casajus, D. 1987 La Tente dans la solitude. La société et les morts chez les Touaregs Kel Ferwan. Paris et Cambridge: Éditions de la Maison des sciences de l'homme et Cambridge University Press.

Claudot-Hawad, H. 1993 Les Touaregs, Portrait en fragments. Aix-en-Provence: Édisud.

Gosselain, O., Zeebroek Decroly, R. \& Decroly, J.-M. 2009 Les Tribulations d'une casserole chinoise au Niger, Techniques \& culture 51: 18-89.

Hincker, C. 2005b Le goût des hommes excellents. Style, esthétique et identité chez les Touaregs de l'Ouest (Mali) in B. Martinelli (dir.) L'Interrogation du style. Anthropologie, techniques et esthétique. Aix-en-Provence: Presses de l'Université de Provence.

- 2005a Le Style touareg ou la fonction sociale des techniques, Aix-en-Provence et Paris: IREMAM et ParisMéditerranée.

Julien, M.-P., Rosselin, C. 2005 La Culture matérielle. Paris: La Découverte (« repères »).

Julien, M.-P., Warnier, J.-P. (dir.) 1999 Approches de la culture matérielle, Corps à corps avec l'objet. Paris: L'Harmattan.

Lemonnier, P. 2000 Technique (système). In P. Bonte \& M. Izard (dir.) Dictionnaire de l'ethnologie et de l'anthropologie. Paris: PUF (« Quadriges »): 697-698.

Lemonnier, P. (dir.) 1993 Technological choices, Transformation in material cultures since the Neolithic. London et New York: Routledge.

Leroi-Gourhan, A. 1992 [1943] L'Homme et la matière. Paris: Albin Michel.

Martinelli, B. (dir.) 2005 L'Interrogation du style, Anthropologie, techniques et esthétique. Aix-en-Provence: Presses de l'Université de Provence.

Nicolaisen, J., Nicolaisen, I. 1997 The Pastoral Tuareg. Ecology, Culture and Society. London: Thames and Hudson.

Prussin, L. 1995 African Nomadic Architecture. Space, Place and Gender. Washington-London: Smithsonian Institution Press and The National Museum of African Art.

Rosselin, C. 2002 L'Observation des pratiques habitantes dans les logements d'une seule pièce, Communications 73 : 95-112.

Warnier, J.-P. 1999 Construire la culture matérielle, L'homme qui pensait avec ses doigts. Paris: PUF. 


\section{RÉSUMÉ}

Habiter ou "faire avec" le sable. Domestiquer l'espace et la matière en contexte saharien. Ce texte explore la relation complexe et dynamique des Sahariens avec le sable et prend l'espace domestique des pasteurs nomades du désert mauritanien comme point d'observation privilégié des interactions entre corps, objets et matière ambiante. Il s'agit de comprendre ce que «faire avec » le sable implique en termes de choix technologiques, de conduites motrices et d'adaptations matérielles au sein de cette cellule familiale minimale qu'est la tente. Si la domestication de cette matière s'inscrit dans un rapport au monde spécifique et est jalonnée de rituels de protection, elle passe surtout par un savoir-vivre sous la tente et un savoir-faire que les individus acquièrent dans leur enfance au contact permanent du sable, mais aussi par la médiation d'objets pensés pour la vie dans ou sur le sable (importance du tissé et des objets à base arrondie). Cette matière ambiante, tantôt solide, tantôt souple voire liquide, donne bien lieu, associée à d'autres exigences comme celle de mobilité, à une culture matérielle spécifique et partagée par l'ensemble de cette société bédouine ouest-saharienne. La sédentarisation et l'urbanisation récentes tendent à brouiller ce style de vie singulier, mettant le sable en concurrence avec ces nouvelles matières que sont notamment le béton et le bitume: valorisé en contexte bédouin pour ses vertus purificatrices, le sable devient souvent indésirable en ville lorsqu'il envahit la maison ou exerce son pouvoir corrosif sur des objets importés sophistiqués; pourtant, il continue d'occuper une place de choix dans l'imaginaire et les pratiques récréatives des citadins.

\section{ABSTRACT}

Living or "dealing with" sand. Domesticating space and matter in a saharan context. This text investigates the complex and dynamic relationship between Saharan people and sand. It takes the domestic space of the nomadic pastoralists of the Mauritanian desert as a privileged place to observe the interactions between bodies, objects and ambient matter. The article aims at understanding what « dealing with » the sand involves in terms of technological choices, motor behaviours and material adaptations within the minimal family unit that is the tent. While the domestication of this matter expresses a specific representation of the environment and implies protection rituals, it especially mobilizes "savoir-vivre" or know-how that people learn in their childhood in everyday contact with the sand, but also through the mediation of objects designed for life in or on the sand (importance of woven artefacts and objects with a rounded base). This ambient matter, either solid or flexible, or even liquid, in association with other requirements such as the need for mobility, gives rise to a specific material culture shared by the north-west Saharan bedouin society. Recent sedentarization and urbanization have tended to erase this particular lifestyle, putting sand in competition with these new materials, for example concrete and asphalt. Appreciated in the bedouin context for its purificatory virtues, sand often becomes unwanted in the city when it invades the house or when it exercises its corrosive power on sophisticated imported objects. Nevertheless, it continues to occupy an important place in the citizen's imagination and in their leisure practices.

\section{MOTS CLÉS}

Sable, tente, confort, objets, Mauritanie, Sahara

\section{KEYWORDS}

Sand, tent, comfort, objects, Mauritania, Sahara 